\title{
Anion photoelectron spectroscopy of protein chromophores
}

\author{
Alice Henley ${ }^{\mathrm{a}}$ and Helen H. Fielding ${ }^{\mathrm{a} *}$

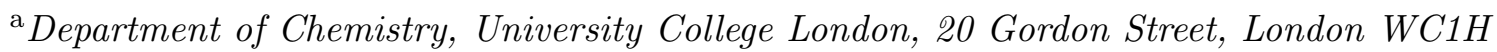 \\ $O A J, U K$
}

(November 12, 2018)

\begin{abstract}
Photoactive proteins that efficiently and selectively transfer light energy into a physical response are ubiquitous in nature. The small molecule chromophores that lie at the heart of these processes often exist as closed-shell anions following deprotonation in proton-transfer reactions. This review highlights the important role that anion photoelectron spectroscopy, combined with computational chemistry calculations, is playing in improving our understanding of the electronic structure and relaxation dynamics of these protein chromophores. We discuss key aspects of anion photoelectron spectroscopy. We then review recent anion photoelectron spectroscopy studies of the deprotonated chromophore anions found in green fluorescent protein (GFP), photoactive yellow protein (PYP) and the deprotonated oxyluciferin anion found in the luciferase enzyme.
\end{abstract}

Keywords: anion photoelectron spectroscopy; photodetachment; electrospray ionisation; green fluorescent protein; photoactive yellow protein; luciferin

\section{Contents}

PAGE

\section{Introduction}

In nature, many organisms have evolved to perform biological processes using photoactive proteins that efficiently and selectively transfer light energy into a physical response. Well known examples include photosynthesis, vision and the production of vitamin $\mathrm{D}$. At the heart of all these processes is a small chromophore that absorbs the light and undergoes small-scale structural changes, which are then captured and amplified by the protein around it and used to initiate a macroscopic response.

When a photoactive protein chromophore absorbs a photon it is promoted from its electronic ground state, $\mathrm{S}_{0}$, to an electronically excited singlet state, $\mathrm{S}_{n}$, in which the nuclei may no longer be in their equilibrium positions. Subsequent redistribution of internal energy may then occur in a number of ways [1]. The coupling between vibrational modes of the chromophore may lead to intramolecular vibrational energy redistribution (IVR), typically on a timescale of $1-100$ ps. The chromophore may then relax from the excited singlet state back to the electronic ground state by emitting a photon, $\mathrm{S}_{n} \rightarrow \mathrm{S}_{0}+h \nu$ (fluorescence), on a timescale of 100 ps $-100 \mathrm{~ns}$. However, ultrafast non-radiative relaxation processes often compete with radiative

\footnotetext{
*Corresponding author. Email: h.h.fielding@ucl.ac.uk

ISSN: print/ISSN online

(C) Taylor \& Francis

DOI:

http://www.tandfonline.com
} 
relaxation; for example, the chromophore can relax from an excited singlet state to a lower lying excited singlet state or the electronic ground state by undergoing internal conversion (IC) on a timescale of approximately 10 ps - 10 fs. Alternatively, the chromophore may undergo intersystem crossing (ISC) from the excited singlet state to a lower lying triplet state, typically on a longer timescale of $100 \mathrm{ps}-10 \mathrm{~ns}$, depending on the strength of the spin-orbit coupling between the singlet and triplet states. The chromophore can then relax back to the electronic ground state from the low lying triplet state by emitting a photon, $\mathrm{T}_{n} \rightarrow \mathrm{S}_{0}+h \nu$ (phosphorescence), on a timescale of $1 \mu \mathrm{s}-1 \mathrm{~s}$, or undergo reverse ISC back to the electronic ground state, typically on a timescale of $1 \mu \mathrm{s}-10 \mathrm{~ms}$ [2].

Many protein chromophores exist as closed-shell anions following deprotonation during proton-transfer reactions within their proteins; examples include the deprotonated chromophore anions that lie at the heart of green fluorescent protein (GFP) $[3,4]$ and photoactive yellow protein (PYP) [5] and the deprotonated oxyluciferin anion in the luciferase enzyme that is responsible for the emission of light from fireflies [6] (Fig. 1). For deprotonated chromophore anions, electron emission is another common relaxation process. Photoinduced electron emission is a ubiquitous phenomenon in biology; a particularly well-known example is the repair mechanism for photodamaged DNA, which involves photoinduced electron transfer from the electronically excited cofactor of DNA photolyase, flavin adenine dinucleotide $\left(\mathrm{FADH}^{-}\right)$[7].
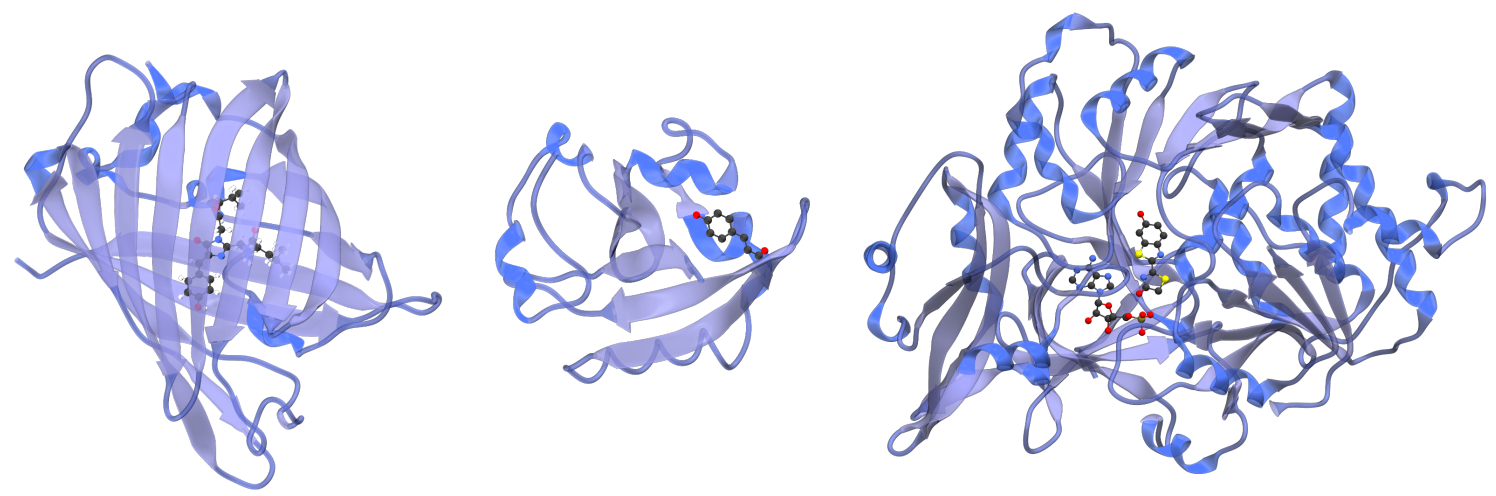

Figure 1. Structures of GFP (left), PYP (centre) and the luciferase enzyme (right) with chromophores enclosed [8].

Understanding the competition between all the possible electronic relaxation processes in a photoactive protein requires a detailed understanding of the electronic structure of the chromophore. Experimentally, the most direct way of determining electronic structure is through the measurement of electron binding energies using photoelectron spectroscopy. Measuring the electron binding energies of isolated protein chromophores in the gas phase, free from interactions with their protein environment, allows the intrinsic electronic structure of the chromophore to be determined in detail. Comparing the electronic structure and dynamics of the isolated chromophore with those of the chromophore in its protein environment then sheds light on the 
role of the interactions with the protein environment. Moreover, gas-phase measurements provide access to the higher lying electronically excited states of the protein chromophore that cannot be measured easily in their native protein environments because the UV absorption of the chromophore overlaps with that of aromatic amino acid residues. The aim of this review is to highlight the important role that photoelectron spectroscopy has played, and continues to play, in improving our understanding of the electronic structure and relaxation dynamics of deprotonated protein chromophore anions in the gas phase.

\section{Methods}

\subsection{Anion photoelectron spectroscopy}

Anion photoelectron spectroscopy records the electron kinetic energy (eKE) distributions following photodetachment of an electron, $\mathrm{e}^{-}$, from a molecular anion. The photodetachment process for a closed-shell molecular anion, $\mathrm{M}^{-}$, is represented as,

$$
\mathrm{M}^{-}+h \nu \rightarrow \mathrm{M}^{\bullet}+\mathrm{e}^{-}
$$

where $\mathrm{M}^{\bullet}$ is the neutral radical formed by removing the electron. In the independent electron approximation, photodetachment occurs without any reorganisation of the remaining electrons (Koopmans' picture [9]) and the eKE distribution of photoelectrons allows us to determine the electron binding energy (eBE) of the molecular orbital from which the electron is detached, with respect to the electronic state of the neutral radical that is left behind, using $\mathrm{eBE}=h \nu-\mathrm{eKE}$. The detachment process is effectively instantaneous compared to the timescale for nuclear rearrangement, so the spectral profile is determined by the Franck-Condon overlap of vibrational wavefunctions of the ground electronic state of the anion and the ground electronic state of the corresponding neutral; for a closed-shell anion, these are $S_{0}$ and $D_{0}$, respectively.

Important quantities are the adiabatic detachment energy (ADE) and vertical detachment energy (VDE), illustrated in Fig. 2. The ADE is the difference between the electronic energies of the anion and corresponding neutral radical, in their ground vibrational states. The VDE is the difference between the electronic energies of the anion and corresponding neutral radical at the geometry of the anion in its ground vibrational state. Experimentally, the ADE and VDE are approximately equivalent to the photon energies corresponding to the onset of the photoelectron spectrum and the maximum in the photoelectron distribution, respectively.

Since the valence electrons of gas-phase molecular anions are only weakly bound, almost all their excited electronic states, $\left[\mathrm{M}^{-}\right]^{*}$, lie above the ADE and are susceptible to indirect, or delayed, electron detachment. As a result, UV photoelectron spectra of anions have features that arise from indirect detachment processes as well as direct detachment processes and these are illustrated in Fig. 3. Direct photodetachment from $\mathrm{S}_{0}$ to the $\mathrm{D}_{0}$ continuum gives rise to electrons with maximum eKE determined by the Franck-Condon overlap of vibrational wavefunctions of $\mathrm{S}_{0}$ and $\mathrm{D}_{0}$, $\epsilon_{1} \sim h \nu-$ VDE. Indirect photodetachment is represented as,

$$
\mathrm{M}^{-}+h \nu \rightarrow\left[\mathrm{M}^{-}\right]^{*} \rightarrow \mathrm{M}^{\bullet}+\mathrm{e}^{-} .
$$

For molecular anions that do not undergo large geometry changes during photodetachment, there is a propensity for conserving vibrational energy (Fig. 3). Thus, indi- 


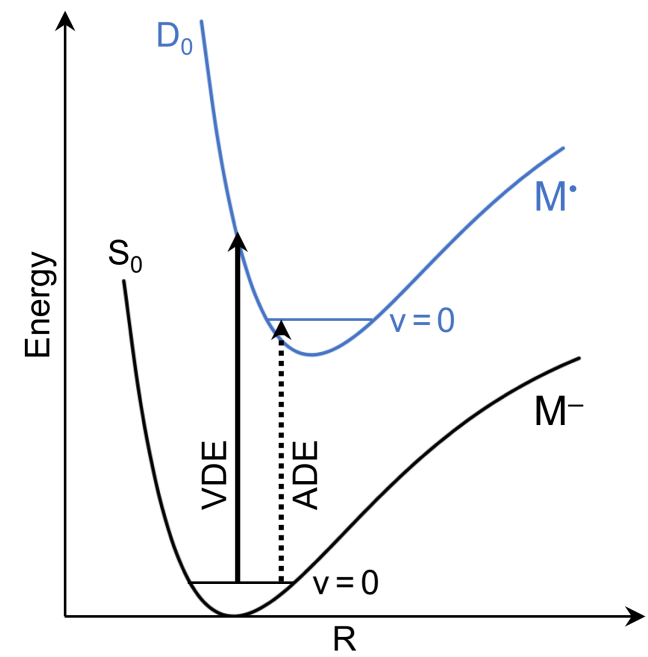

Figure 2. A schematic illustration of the potential energy curves for a molecular anion, $\mathrm{M}^{-}$, in its closed shell ground electronic state, $\mathrm{S}_{0}$, and the corresponding neutral radical, $\mathrm{M}^{*}$, in its ground electronic state, $\mathrm{D}_{0}$, as a function of a single vibrational mode coordinate, $R$. Vertical arrows represent the experimental vertical detachment energy (VDE) and adiabatic detachment energy (ADE).

rect photodetachment following photoexcitation of $\mathrm{S}_{2}$ with excess vibrational energy, $E_{\mathrm{v}}=h \nu-E\left(\mathrm{~S}_{2}\right)$, where $E\left(\mathrm{~S}_{2}\right)$ is the adiabatic excitation energy of $\mathrm{S}_{2}$, will result in the emission of photoelectrons with eKE, $\epsilon_{2} \sim h \nu-E\left(\mathrm{D}_{0}\right)-E_{\mathrm{v}}$, where $E\left(\mathrm{D}_{0}\right)$ is the $\mathrm{ADE}$. This last expression can be rewritten as, $\epsilon_{2} \sim E\left(\mathrm{~S}_{2}\right)-E\left(\mathrm{D}_{0}\right)$; i.e. the photoelectrons are emitted with eKE corresponding to the $\mathrm{S}_{2}-\mathrm{D}_{0}$ energy difference. Alternatively, photoexcitation of $\mathrm{S}_{2}$ may be followed by rapid IC to $\mathrm{S}_{1}$ with excess vibrational energy $E_{\mathrm{v}}=h \nu-E\left(\mathrm{~S}_{1}\right)$, where $E\left(\mathrm{~S}_{1}\right)$ is the adiabatic excitation energy of $\mathrm{S}_{1}$, and the emission of photoelectrons with very low eKE, $\epsilon_{3} \sim 0$. If the anion undergoes IC back to $\mathrm{S}_{0}$, vibration-electronic coupling between the ground electronic states of the anion and neutral radical may result in vibrational autodetachment [10]. Alternatvely, coupling between the very high density of vibrational states with energy, $E_{\mathrm{v}}=h \nu$, may result in statistical redistribution of vibrational energy and a delayed emission of electrons with an eKE profile, $P(\epsilon)$, defined by Klots' formula $[11,12]$,

$$
P(\epsilon) \propto \epsilon^{1 / 2} \exp \left(-\epsilon / k_{\mathrm{B}} T_{\mathrm{M}^{*}}\right),
$$

where $k_{\mathrm{B}}$ is Boltzmann's constant and $T_{\mathrm{M}}$ is the temperature of the neutral radical [13-15],

$$
T_{\mathrm{M}^{\bullet}}=T_{\mathrm{M}^{-}}+(h \nu-\mathrm{ADE}) / C_{\mathrm{v}} .
$$

$T_{\mathrm{M}^{-}}$is the microcanonical temperature of the anions before photoexcitation and $C_{\mathrm{v}}$ is the microcanonical heat capacity.

Direct photodetachment and indirect photodetachment processes can be identified by monitoring how features in photoelectron spectra, plotted as functions of eKE and eBE, vary with photon energy (Fig. 4). Direct photodetachment from the ground electronic state of the anion, $\mathrm{S}_{0}$, to the ground electronic state of the neutral radical, 


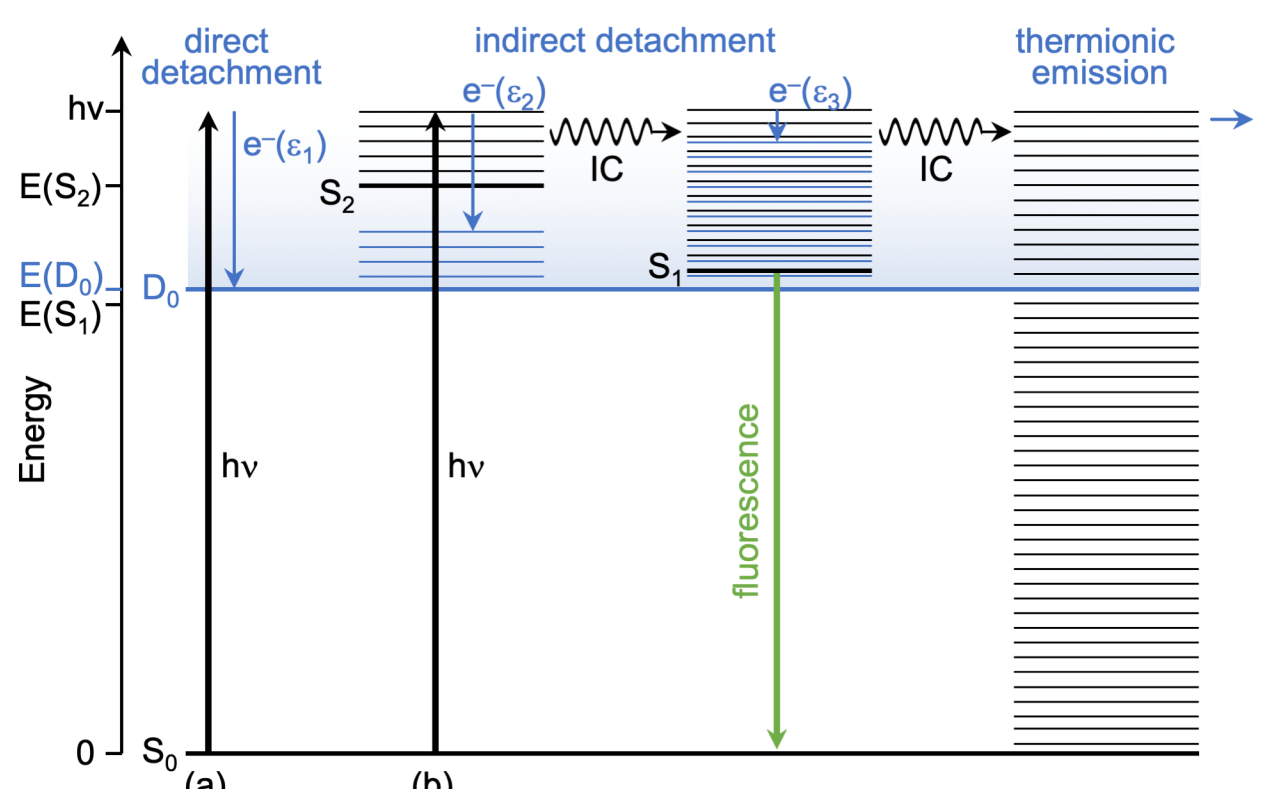

Figure 3. Schematic energy level diagram illustrating UV photoelectron spectroscopy of an anion. Thin horizontal black lines represent the vibrational levels of the electronic states of the anion and the blue shaded area represents the electron detachment continuum. Vertical blue arrows represent the eKE of direct and indirect electron detachment processes and the thin horizontal blue lines represent the vibrational energy left in the neutral radical following electron detachment (determined by the propensity for conservation of vibrational energy). The horizontal black arrows represent some of the possible internal conversion (IC) processes and the horizontal blue arrow represents thermionic emission (TE). (a) Direct photodetachment from $\mathrm{S}_{0}$ to the $\mathrm{D}_{0}$ continuum gives electrons with eKE, $\epsilon_{1} \sim h \nu-\mathrm{VDE}$; in this particular example, VDE $=\mathrm{ADE}$. (b) Indirect photodetachment following photoexcitation of $\mathrm{S}_{2}$ with excess vibrational energy, $E_{\mathrm{v}}=h \nu-E\left(\mathrm{~S}_{2}\right)$ gives electrons with eKE, $\epsilon_{2} \sim E\left(\mathrm{~S}_{2}\right)-E\left(\mathrm{D}_{0}\right)$. Indirect photodetachment following photoexcitation of $\mathrm{S}_{2}$ and subsequent IC to $\mathrm{S}_{1}$ gives electrons with eKE, $\epsilon_{3} \sim 0$. TE from $\mathrm{S}_{0}$ following photoexcitation of $\mathrm{S}_{2}$ and subsequent IC back to $\mathrm{S}_{0}$ gives electrons with an eKE distribution profile defined by Eq. (3).

$\mathrm{D}_{0}$, gives rise to a photoelectron distribution whose eKE increases linearly with photon energy but whose eBE is constant and corresponds to the $\mathrm{S}_{0}-\mathrm{D}_{0}$ VDE. Indirect photodetachment from a resonance in the detachment continuum, $\mathrm{S}_{n}$, gives rise to a photoelectron distribution whose eKE remains constant with increasing photon energy and corresponds to the energy difference, $E\left(\mathrm{~S}_{n}\right)-E\left(\mathrm{D}_{0}\right)$. Thermionic emission from hot $\mathrm{S}_{0}$, populated by IC from an electronically excited state of the anion, is characterised by a photoelectron distribution with a profile defined by Eq. (3) with $\mathrm{eKE} \sim 0(\mathrm{eBE} \sim h \nu)$

The competition between indirect detachment and internal conversion is governed by their timescales. The timescale for indirect electron detachment is typically in the range $10 \mathrm{fs}-10 \mathrm{ps}$, depending on the strength of the coupling between the electronically excited state and the continuum. Excited electronic states embedded in the detachment continuum are classified either as shape or Feshbach resonances (Fig. 5). Shape resonances only require the loss of a single electron to generate the neutral 

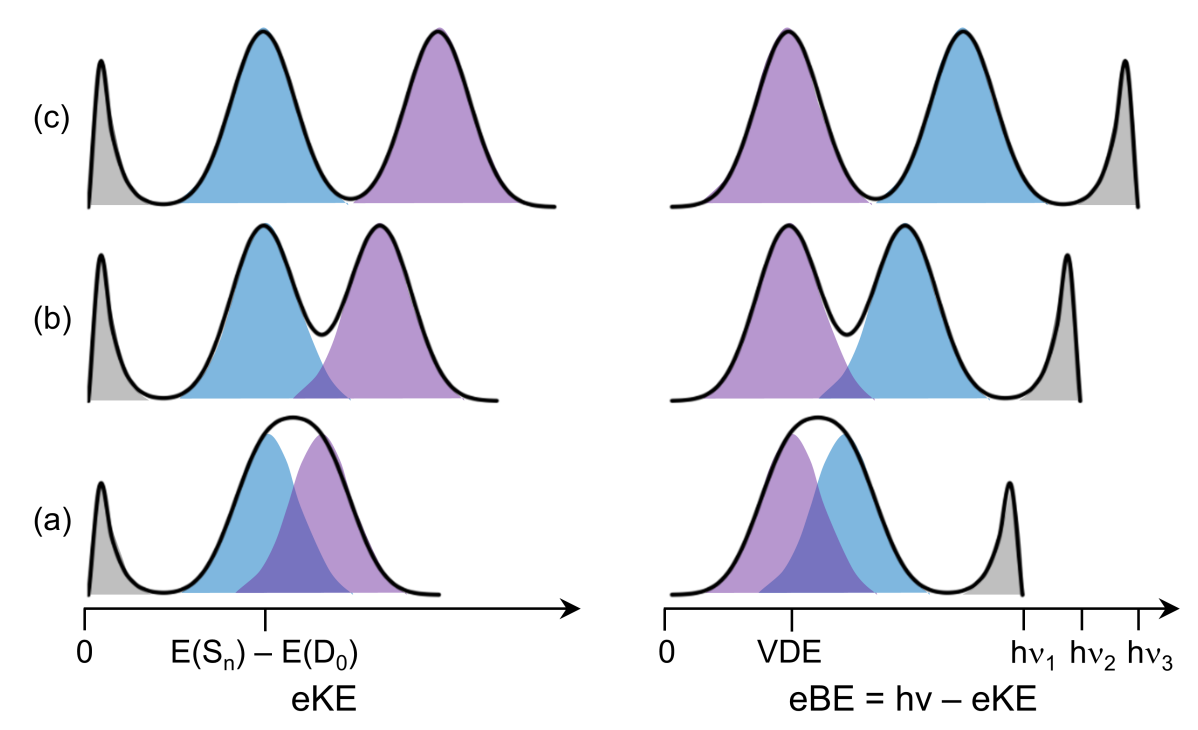

Figure 4. Cartoon illustrating how features corresponding to direct (purple) and indirect (blue, grey) photodetachment processes manifest themselves in photoelectron spectra plotted as functions of eKE (left) and eBE (right) for photon energies, (a) $h \nu_{1}$, (b) $h \nu_{2}$ and (c) $h \nu_{3}$, where $h \nu_{1}<h \nu_{2}<$ $h \nu_{3}$. Direct photodetachment from $\mathrm{S}_{0}$ to $\mathrm{D}_{0}$ gives rise to photoelectrons with a maximum eBE that corresponds to the $\mathrm{S}_{0}-\mathrm{D}_{0} \mathrm{VDE}$ (purple). Indirect photodetachment following photoexcitation of a resonance in the detachment continuum, $\mathrm{S}_{n}$, gives rise to photoelectrons with a maximum eKE corresponding to $E\left(\mathrm{~S}_{n}\right)-E\left(\mathrm{D}_{0}\right)$ (blue). TE from $\mathrm{S}_{0}$ following photoexcitation of an electronically excited state of the anion and subsequent IC back to $S_{0}$ gives electrons with a characteristic energy distribution profile defined by Eq. (3) (grey).

electron configuration, whereas Feshbach resonances require concerted electronic reconfiguration and loss of an electron to generate the neutral electron configuration. Consequently, electronically excited states with shape resonance character are coupled more strongly to the detachment continuum than those with Feshbach resonance character and, therefore, electron detachment from a shape resonance is much faster than that from a Feshbach resonance. Electron detachment from an excited-shape resonance (Fig. 5) is even faster.

\subsection{Time-resolved anion photoelectron spectroscopy}

In a time-resolved anion photoelectron spectroscopy (TRPES) experiment, an ultrashort pump laser pulse (typically $\sim 100 \mathrm{fs}$ ) promotes an anion to an excited electronic state and a second ultrashort probe laser pulse then detaches an electron from the evolving excited state of the anion. The photoelectron spectra are then measured at a series of precisely timed pump-probe time intervals. One of the advantages of TRPES over other pump-probe methods, such as transient absorption spectroscopy or timeresolved fluorescence spectroscopy, is that electron detachment is always an allowed process because there are no stringent selection rules for photodetachment. However, it should be noted that the detachment cross-section, $\sigma \propto \mathrm{eKE}^{l+1 / 2}[16,17]$, where $l$ is the orbital angular momentum of the outgoing electron wave, shows that $\sigma \rightarrow 0$ as 


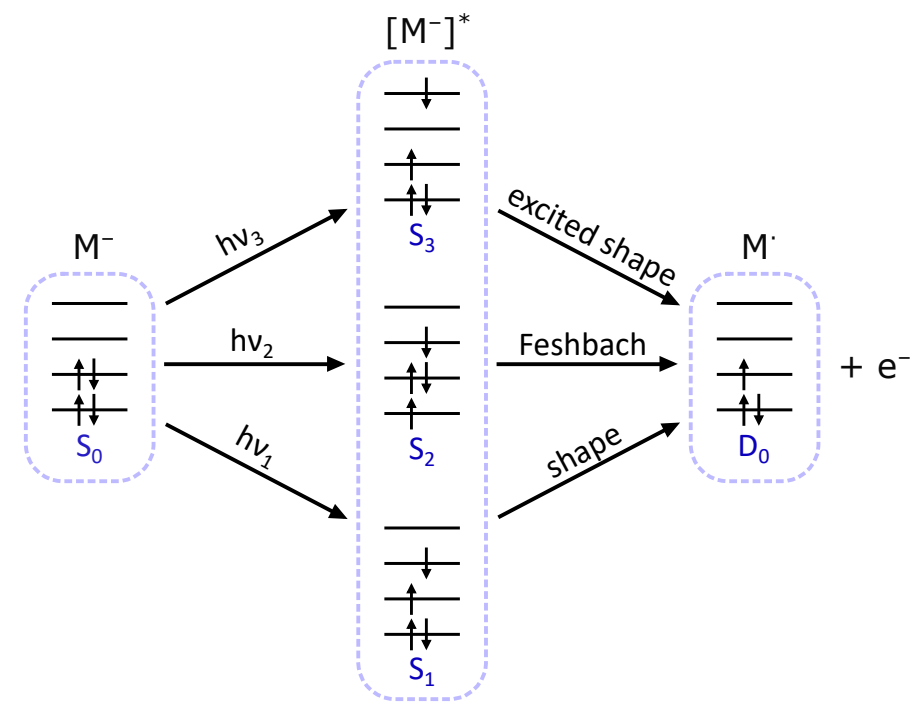

Figure 5. Electron configurations of electronically excited states of an anion, $\left[\mathrm{M}^{-}\right]^{*}$, with shape, Feshbach and excited-shape resonance characters with respect to the detachment continuum associated with the neutral radical, $\mathrm{M}^{*}$, in its electronic ground-state, $\mathrm{D}_{0}$.

$l \rightarrow 0$. One of the advantages of anion TRPES over neutral-molecule TRPES is that the relatively small photodetachment energies of only a few $\mathrm{eV}$ make it relatively straightforward to monitor repopulation of the ground state of the anion using UV laser pulses.

\subsection{Experimental considerations}

Although there are many ways to generate gas-phase molecular anions, electrospray ionisation (ESI) has proved to be a particularly valuable tool for transferring deprotonated molecular anions into the gas phase in the same closed-shell electronic ground states that exist is nature, and is the method employed in our group [18]. ESI produces the gaseous ions from sample solutions flowing through a metal capillary maintained at high voltage and atmospheric pressure. The ions move under the influence of the electric field causing charge to build at the capillary tip. Eventually, repulsion between the ions overcomes the surface tension of the liquid and the surface expands until, if the applied field is high enough, a fine spray of highly charged droplets emerges. Solvent in the droplets evaporates resulting in 'Coulomb explosions' and droplet fission. The resulting small droplets then evaporate further, eventually leading to the production of isolated gas-phase ions. ESI has been combined very successfully with photoelectron spectroscopy for studying the electronic structure of a wide range of deprotonated biomolecules, including protein chromophores [19-35].

There are several techniques for measuring photoelectron spectra, the most popular of which are based on time-of-flight (TOF) or velocity map imaging (VMI) methods. TOF spectrometers measure the eKE distribution by analysing the time taken for the electrons to travel from the laser-molecule interaction region to an electron detec- 
tor. A commonly employed TOF spectrometer is the magnetic bottle photoelectron spectrometer in which photoelectrons created in the laser-molecule interaction region are guided in a magnetic field toward the electron detector, which is usually a microchannel plate [36]. VMI uses static electric fields to project the 3D photoelectron momentum distribution created in the laser-molecule interaction region onto a $2 \mathrm{D}$ position-sensitive electron detector, which usually comprises a microchannel plate, a phosphor screen and a charge-coupled device (CCD) camera [37]. The original 3D photoelectron momentum distribution can then be recovered from the $2 \mathrm{D}$ image using using well-established methods.

VMI allows the photoelectron angular distribution (PAD) to be measured as well as the eKE distribution, which provides additional information about the molecular orbital from which the electron is detached [38]. As an electron leaves a molecular anion, its wave function reflects the electronic and nuclear configurations of the anion at the moment of electron detachment. There is a requirement that the direct product of the irreducible representations of the free electron wave function, $\Gamma_{\mathrm{e}^{-}}$, the electronic state of the neutral radical, $\Gamma_{\mathrm{M}^{*}}$, the transition dipole moment operator, $\Gamma_{\mu}$, and the electronic state of the molecular anion, $\Gamma_{\mathrm{M}^{-}}$, contains the totally symmetric irreducible representation of the molecular point group, $\Gamma_{\mathrm{s}}$, i.e. $\Gamma_{\mathrm{e}^{-}} \otimes \Gamma_{\mathrm{M}} \cdot \otimes \Gamma_{\mu} \otimes$ $\Gamma_{\mathrm{M}^{-}} \subset \Gamma_{\mathrm{s}}$. The PAD is measured in the laboratory frame relative to the electric field vector of the photoionising laser and reflects the electronic symmetry of the anion and the symmetries of the contributing molecular frame transition dipole moment components. Since the relative contributions of the molecular frame transition dipole moments are determined geometrically by the orientation of the molecular anion relative to the ionising laser field polarisation, the form of the measured PAD will reflect the distribution of molecular anion axes. For a one-photon detachment process (Fig. 3(a)), the PAD can be expressed as

$$
I(\theta) \propto 1+\beta_{2} P_{2}(\cos \theta),
$$

where $I(\theta)$ is the probability of photoelectron emission at a particular angle $\theta$, defined as the angle between the laser polarisation and the velocity vector of the photoelectron, $P_{2}(\cos \theta)$ is the 2 nd order Legendre Polynomial and $\beta_{2}$ is the asymmetry parameter. The two limiting values of $\beta_{2}$ are +2 and -1 , corresponding to photoelectron emission predominantly parallel $\left(\cos ^{2} \theta\right.$ distribution) and perpendicular $\left(\sin ^{2} \theta\right.$ distribution) to the laser polarisation, respectively. In the absence of continuum resonances, photoelectrons with different anisotropies can be assumed to arise from detachment from anion molecular orbitals of different electronic character and, in general, a negative $\beta_{2}$ can be attributed to photodetachment from an orbital with $p$ or $\pi$ character and a positive $\beta_{2}$ can be attributed to photoionisation from an orbital with $s$ or $\sigma$ character. PADs can be very sensitive to continuum resonances and molecular conformations and thus provide an informative additional probe of electronic and molecular structure [39, 40].

\subsection{Computational chemistry considerations}

The interpretation of anion photoelectron spectroscopy measurements frequently requires calculations to determine the ground electronic state of the anion, VDEs, ADEs 
and vertical excitation energies (VEEs). Quantum chemistry calculations of molecular anions are challenging: their relatively small photodetachment energies require accurate calculations to obtain meaningful values; their radially diffuse electron densities require atomic orbital basis sets capable of describing diffuse densities; almost all their electronically excited states lie in the detachment continuum so the coupling between the quasi-bound valence state and the continuum must be considered.

The starting point of any calculation is to determine the deprotonation site and optimise the geometry. Although there are many methods available, we have found that DFT methods work well for deprotonated protein chromophore anions, for example, using B3LYP [41-44] or CAM-B3LYP [45] functionals with double- or triple- $\zeta$ basis sets, polarisation functions and diffuse functions (Pople 6-311++G(3df,3dp) [46-48] or Dunning aug-cc-pVDZ/pVTZ [49]). Frequency calculations confirm that minima in the potential energy surfaces have been reached.

VDEs may be calculated using various methods. We have used the electron propagator theory (EPT) method with the outer valence Greens function (OVGF) propagator $[50,51]$, the equation-of-motion coupled-cluster method with single and double excitation for the calculation of ionisation potentials (EOM-IP-CCSD) [52] and DFT. EPT and EOM-IP-CCSD methods allow VDEs to electronically excited states of the neutral radical to be determined as well as those for the electronic ground-state of the neutral radical. DFT can be used to calculate the VDE to the electronic ground-state of the neutral radical as the energy difference between the anion and neutral radical, at the optimised geometry of the anion, taking zero-point energies into consideration (Fig. 2). It is worth noting that we have found that calculating the energy difference between the anion and neutral radical at the optimised geometry of the anion (without taking into account zero-point energies) yields very similar values for the VDE (within $0.02 \mathrm{eV}$ or $<1 \%$ ). Using DFT to calculate the VDE allows a direct comparison with the DFT calculated ADE, determined as the difference between the lowest vibrational state of the anion and the lowest vibrational state of the neutral radical (the 0-0 transition). Of course higher-level theory has also been used very effectively, for example, XMCQDPT2 [31] and MS-CASPT2 [53] methods.

Table 1 lists VDEs and ADEs calculated using some of the more straightfoward methods, alongside approximate experimental VDEs, for a family of PYP chromophore anions (Fig. 23 in Section 4.5). In terms of the calculated $\mathrm{D}_{0}$ VDEs, the EPT method has determined the VDEs of $p \mathrm{CA}^{-}, p \mathrm{CE}^{-}$and $\mathrm{P} p \mathrm{CE}^{-}$to be just slightly higher than the experimental maxima $(\sim 0.05 \mathrm{eV})$ and slightly lower for $\mathrm{N} p \mathrm{CE}^{-}$ $(0.11 \mathrm{eV})$. The EPT $/ 6-311++\mathrm{G}(3 \mathrm{df}, 3 \mathrm{pd})$ value is within $2 \%$ of the experimental maxima for $p \mathrm{CA}^{-}, p \mathrm{CE}^{-}$and $\mathrm{P} p \mathrm{CE}^{-}$, and $4 \%$ difference of that for $\mathrm{N} p \mathrm{CE}^{-}$. The VDEs calculated using DFT are systematically higher $(\sim 0.15 \mathrm{eV})$ than the experimental maxima. The EOM-IP-CCSD calculations have predicted the VDEs of the chromophores to within $\sim 0.06 \mathrm{eV}$ of the experimental maxima, although the deviations are less systematic than with DFT; for example, the EOM-IP-CCSD VDE of $p \mathrm{CA}^{-}$is higher than the experimental maximum whereas the $\mathrm{VDE}$ of $p \mathrm{CE}^{-}$is lower. It should be noted that the accuracy of these measurements was $\pm 0.05 \mathrm{eV}$ and that the high density of vibrational transitions contributing to these vibrationally unresolved photoelectron spectra can easily shift the maxima away from the true 
VDE. We conclude that all these methods can give VDEs that are in good agreement with experiment and note that even the simple, computationally inexpensive, DFT method performs well.

Table 1. VDEs, B3LYP/6-311++G(3df,3pd) $\mathrm{D}_{0}-\mathrm{S}_{0}$ ADEs and maxima of experimental $346 \mathrm{~nm}$ photoelectron spectra (in eV) for model PYP chromophores $p \mathrm{CA}^{-}, p \mathrm{CE}^{-}, \mathrm{P} p \mathrm{CE}^{-}$and $\mathrm{N} p \mathrm{CE}^{-}$ (Fig. 23). VDEs were calculated using EPT/6-311++G(3df,3pd), EOM-IP-CCSD/aug-cc-pVDZ and B3LYP/6-311++G(3df,3pd) taking zero-point energies into consideration. EPT and EOM-IPCCSD have two values listed corresponding to detachment to $\mathrm{D}_{0}$ and $\mathrm{D}_{1}$ states of the corresponding neutral radicals. Geometries were optimised using the B3LYP/6-311++G(3df,3pd) method. Taken from Ref. [29].

\begin{tabular}{|c|c|c|c|c|c|}
\hline & \multicolumn{3}{|c|}{ VDE } & \multirow{2}{*}{$\begin{array}{l}\text { ADE } \\
(0-0)\end{array}$} & \multirow{2}{*}{ Experiment } \\
\hline & EPT & EOM-IP-CCSD & DFT & & \\
\hline$p \mathrm{CA}^{-}$ & 2.99 & 2.97 & 3.10 & 2.99 & $2.92 \pm 0.05$ \\
\hline$p \mathrm{CE}^{-}$ & $\begin{array}{l}2.91 \\
5.18\end{array}$ & $\begin{array}{l}2.78 \\
4.42\end{array}$ & 3.01 & 2.91 & $2.85 \pm 0.05$ \\
\hline $\mathrm{P} p \mathrm{CE}^{-}$ & $\begin{array}{l}2.96 \\
5.23\end{array}$ & $\begin{array}{l}2.83 \\
4.48\end{array}$ & 3.03 & 2.93 & $2.89 \pm 0.05$ \\
\hline $\mathrm{N} p \mathrm{CE}^{-}$ & $\begin{array}{l}2.67 \\
5.02\end{array}$ & $\begin{array}{l}2.71 \\
4.33\end{array}$ & 2.93 & 2.82 & $2.78 \pm 0.05$ \\
\hline
\end{tabular}

VEEs may be calculated using various methods. The more straightforward approaches include DFT (TD-DFT) [54] and $\mathrm{ADC}(2)[55,56]$ methods. We have found that the simpler TD-DFT method tends to yield VEEs that are consistently around $0.5 \mathrm{eV}$ higher than those determined using higher level methods [23, 25, 28, 29, 34, 35], but that the $\mathrm{ADC}(2)$ method compares favourably with high-level calculations, for example, with SA-CASSCF (14,12)-PT2/cc-pVDZ calculations [57] for a series of PYP chromophore anions (Table 2) [29], and XMCQDPT2/aug-cc-pVTZ level calculations [31] for the GFP chromophore anion [33]. However, due to the number of diffuse functions required in the basis set to obtain an accurate VEE, a huge number of continuum states are calculated. Thus, to assist with the interpretation of $\operatorname{ADC}(2)$ calculations, one can start with a relatively small basis set without diffuse functions, for example, 6-311G(2d,2p), which will not necessarily give accurate VEEs but will allow the nature of the molecular orbitals involved in the transitions to be determined. Then, having identified the key molecular orbitals, the diffuse functions can be added to obtain more accurate VEEs. Improving quantum chemistry approaches to calculating molecular anion resonances in continua is currently a hot topic [58].

For vibrationally-resolved photoelectron spectra, it is sometimes useful to calculate the Franck-Condon overlap in order to identify key vibrational modes. There are many methods, but we have found ezSpectrum [60] to be particularly straightforward $[23,25,35]$. These calculations require the equilibrium geometries, harmonic frequencies and normal mode vectors of the deprotonated anions and corresponding neutral radicals as input. PADs can also be calculated using, for example, ezDyson [61]. The Dyson orbitals required as input can be obtained from EPT or EOM-IP-CCSD calculations and represent the overlap between the $N$ electron molecular wave-function of the anion in its ground electronic state and the $N-1$ electron molecular wavefunction 
Table 2. VEEs calculated using CAM-B3LYP/6-311++G(3df,3pd), ADC(2)/6-31+G** and SACASSCF (14,12)-PT2/cc-pVDZ [57] methods for model PYP chromophores $p \mathrm{CA}^{-}, p \mathrm{CE}^{-}, \mathrm{P} p \mathrm{CE}^{-}$ and $\mathrm{N} p \mathrm{CE}^{-}$(Fig. 23) and experimental values [59]. VEEs are given in $\mathrm{eV}$ and oscillator strengths are shown in brackets. Geometries were optimised using the B3LYP/6-311++G(3df,3pd) method. Taken from Ref. [29].

\begin{tabular}{lllllll}
\hline & & & \multicolumn{1}{c}{$p \mathrm{CA}^{-}$} & $p \mathrm{CE}^{-}$ & $\mathrm{P} p \mathrm{CE}^{-}$ & $\mathrm{N} p \mathrm{CE}^{-}$ \\
\hline CAM-B3LYP & $1^{1} \pi \pi^{*}$ & $\mathrm{~S}_{1}$ & $3.45(0.91)$ & $3.43(0.95)$ & $3.49(0.95)$ & $3.36(0.81)$ \\
& $1^{1} n \pi^{*}$ & $\mathrm{~S}_{2}$ & $4.10(0.00)$ & $4.12(0.00)$ & $4.15(0.00)$ & $4.10(0.00)$ \\
& $2^{1} \pi \pi^{*}$ & $\mathrm{~S}_{3}$ & $4.33(0.07)$ & $4.31(0.11)$ & $4.34(0.06)$ & $4.40(0.03)$ \\
\hline ADC(2) & $1^{1} \pi \pi^{*}$ & $\mathrm{~S}_{1}$ & $2.96(1.00)$ & $2.96(1.02)$ & $3.02(1.03)$ & $2.82(0.88)$ \\
& $1^{1} n \pi^{*}$ & $\mathrm{~S}_{2}$ & $3.35(0.00)$ & $3.37(0.00)$ & $3.39(0.00)$ & $3.26(0.00)$ \\
& $2^{1} \pi \pi^{*}$ & $\mathrm{~S}_{3}$ & $4.13(0.09)$ & $4.10(0.10)$ & $4.10(0.06)$ & $3.94(0.07)$ \\
\hline SA-CASSCF & $1^{1} \pi \pi^{*}$ & $\mathrm{~S}_{1}$ & 2.96 & 2.94 & & \\
$(14,12)-\mathrm{PT} 2$ & $1^{1} n \pi^{*}$ & $\mathrm{~S}_{2}$ & 3.65 & 3.65 & & \\
& $2^{1} \pi \pi^{*}$ & $\mathrm{~S}_{3}$ & 3.82 & 3.82 & &
\end{tabular}

of the corresponding neutral radical $[62,63]$.

\section{Green fluorescent protein chromophores}

\subsection{Background}

Green fluorescent protein (GFP), together with its family of variants, is the most widely used fluorescent protein for in vivo monitoring of biological and biochemical processes $[3,4,64-66]$. The chromophore that lies at the heart of GFP is 4-hydroxybenzylidene-1,2-dimethylimidazolinone ( $p$-HBDI); the deprotonated anionic form, $p$ $\mathrm{HBDI}^{-}$, is shown in Fig. 6.

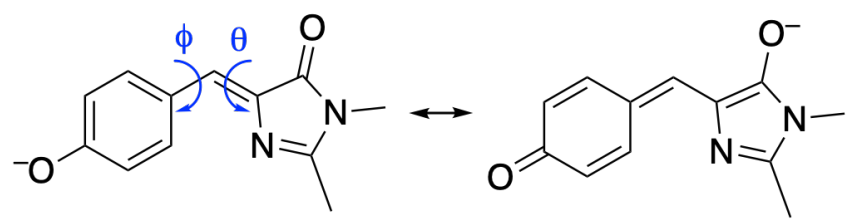

Figure 6. The two resonance forms of the deprotonated GFP chromophore anion, $p$-HBDI ${ }^{-}$, showing torsional angles $\phi$ and $\theta$ referred to later in the text and in Fig. 10.

In GFP, the chromophore is anchored both covalently and via a hydrogen-bonded network to the protein that is wrapped around it in a $\beta$-barrel structure (Fig. 1). GFP has two absorption bands, centred around $395 \mathrm{~nm}$ and $480 \mathrm{~nm}$, that are attributed to the neutral and anionic forms, respectively. Following excitation of the neutral form of the chromophore, excited-state proton transfer takes place via a proton relay between water molecules and amino acid residues, followed by fluorescence (with quantum yield, $\Phi \approx 0.8$ ) from the deprotonated anionic form of the chromophore [67]. The fluorescence of GFP is lost when the protein is denatured, but 
it is recovered when the protein refolds [68]. Furthermore, the isolated chromophore is virtually non-fluorescent at biological temperatures in solution [69] and in vacuo [70], but it fluoresces strongly in solution when cooled to $77 \mathrm{~K}$ [69] and it has been found to have a sufficiently long excited state lifetime in the gas phase when cooled to $100 \mathrm{~K}$ that fluorescence may occur in vacuo at low temperatures [71]. The isolated chromophore can also become fluorescent when incorporated in rigid scaffolds, such as metal-organic frameworks or other non-native protein environments [72-75]. The lack of fluorescence from the free chromophore at biological temperatures is attributed to ultrafast isomerisation around the central C-C-C bridge linking the phenolate and imidazolinone moieties (Fig. 6), followed by efficient internal conversion to take excited state population back to the electronic ground state $[76,77]$. When the molecular framework of the chromophore is held rigid by the protein or a rigid scaffold, isomerisation around the $\mathrm{C}-\mathrm{C}-\mathrm{C}$ bridge is impeded and fluorescence from the excited state becomes the primary decay channel. Numerous spectroscopic and computational chemistry studies of the electronic structure of the isolated GFP protein chromophore have been aimed at unravelling the mechanism of competing internal conversion and the role of the chromophore environment in enhancing and tuning the fluorescent properties of GFPs (for a comprehensive recent review see $[78])$.

\subsection{Vertical detachment energy}

The first experimental study of the electronic structure of gas-phase $p$-HBDI ${ }^{-}$combined ESI with the electrostatic ion storage ring in Aarhus, ELISA, to record an action-absorption spectrum of the GFP chromophore [79]. The action-absorption band was found to have a maximum at $479 \mathrm{~nm}(2.59 \mathrm{eV})$, which is very close to the absorption maximum of the anionic form of the protein $(480 \mathrm{~nm})$; this led to the suggestion that the $\beta$-barrel structure provided an electronic environment that was similar to a vacuum. Electronic structure calculations reported by Krylov and coworkers calculated a vertical excitation energy (VEE) for $\mathrm{S}_{1}$ that was in excellent agreement with the action spectroscopy maximum $(2.6 \mathrm{eV})$ [80]. These early calculations also reported VDEs in the range 2.4-2.5 eV, suggesting that $\mathrm{S}_{1}$ was a resonance in the detachment continuum. Subsequent action-absorption measurements reported by Forbes and Jockusch showed that, in addition to fragmentation, electron detachment was a significant relaxation pathway in gas-phase action-absorption measurements [70].

The photodetachment process was then measured directly in three independent photoelectron spectroscopy studies that found the VDE to lie in the range 2.68$2.85 \mathrm{eV}$ [19-21]. Importantly, these measurements showed that the $\mathrm{S}_{1}$ state was bound with respect to photodetachment in the Franck-Condon region; however, the higher lying vibrational states of $\mathrm{S}_{1}$ lie in the $\mathrm{D}_{0}$ detachment continuum and, since $\mathrm{S}_{1}$ has shape resonance character with respect to $\mathrm{D}_{0}$, vibrational modes that couple $\mathrm{S}_{1}$ and $\mathrm{D}_{0}$ can undergo efficient vibrational autodetachment. Several groups have also used a range of computational methods to calculate VDEs that are close to these experimental measurements [21, 23, 81]. A recent photoelectron spectrum from 
our group is presented in Fig. 7 alongside a vibrationally-resolved low-temperature photoelectron spectrum of $p$-HBDI ${ }^{-}$, from which the ADE has been determined to be $2.73 \pm 0.01 \mathrm{eV}[24]$, and a spectrum calculated using the XMCQDPT2/aug-cc-pVTZ method [31].

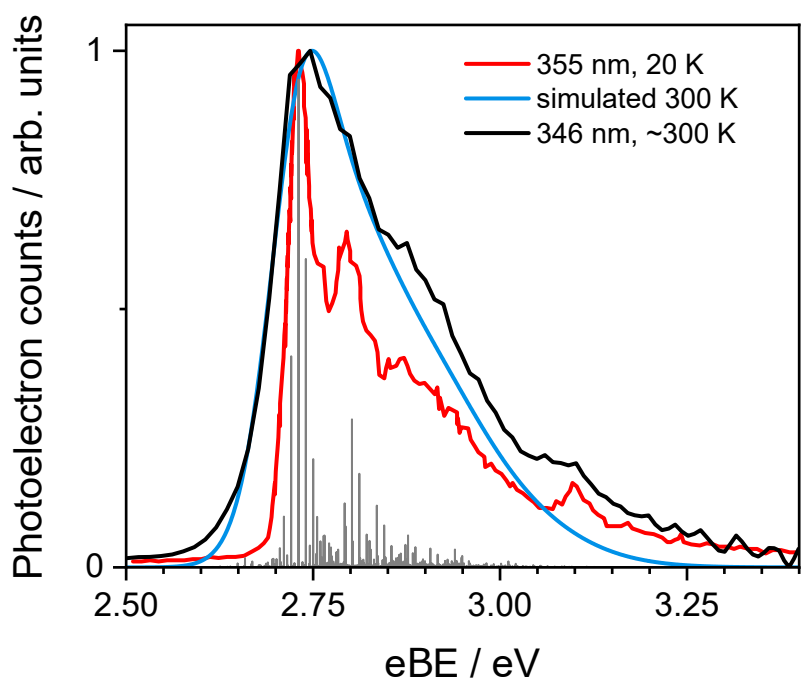

Figure 7. Photoelectron spectra of $p$-HBDI ${ }^{-}$. Black line: $346 \mathrm{~nm}$ photoelectron spectrum of $p \mathrm{HBDI}^{-}(\sim 300 \mathrm{~K})$ recorded recently by our group. Red line: low-temperature $355 \mathrm{~nm}$ photoelectron spectrum of $p \mathrm{HBDI}^{-}(20 \mathrm{~K})$ reported by Wang and coworkers [24]. Adapted with permission from [24]. Copyright 2014 American Chemical Society. XMCQDPT2/aug-cc-pVTZ calculated $\mathrm{S}_{0}-\mathrm{D}_{0}$ stick spectrum at $300 \mathrm{~K}$ [31]. Reproduced from Ref. [31] with permission from the Royal Society of Chemistry. Grey line: stick spectrum convolved with the Gaussian instrument function of our experimental spectrum $(\Delta E / E=5.4 \%$ at $0.5 \mathrm{eV}$ eKE).

\section{3. $\mathrm{S}_{1}$ dynamics}

The timescales associated with the electronic relaxation of $p$-HBDI ${ }^{-}$in the gas phase following photoexcitation of the $\mathrm{S}_{1}$ state have been determined using TRPES [22]. Gas-phase anions were excited with $500 \mathrm{~nm}(2.48 \mathrm{eV})$ femtosecond pump pulses, close to the maximum of the $\mathrm{S}_{1}-\mathrm{S}_{0}$ absorption profile [79] but below the ADE [24]. The evolution of population on the excited state was monitored by firing $800 \mathrm{~nm}$ $(1.55 \mathrm{eV})$ femtosecond probe pulses at a series of pump-probe delays to project the excited state population onto the photodetachment continuum (Fig. 8).

At $t=0$, the photoelectron spectrum was observed to have a maximum eKE around $1.1 \mathrm{eV}$ (Fig. 9). With increasing pump-probe delay, the high eKE component of the photoelectron spectrum was observed to decay on a sub-picosecond timescale, leaving a broad photoelectron spectrum centred around $0.6 \mathrm{eV}$ eKE, which subsequently decayed on a picosecond timescale. The TRPES were fit to a sum of exponentially decaying profiles convoluted with a Gaussian instrument function [82] from which it was determined that the excited state dynamics were dominated by processes with two distinct timescales, $\tau_{1}=330$ fs and $\tau_{2}=1.4$ ps. 


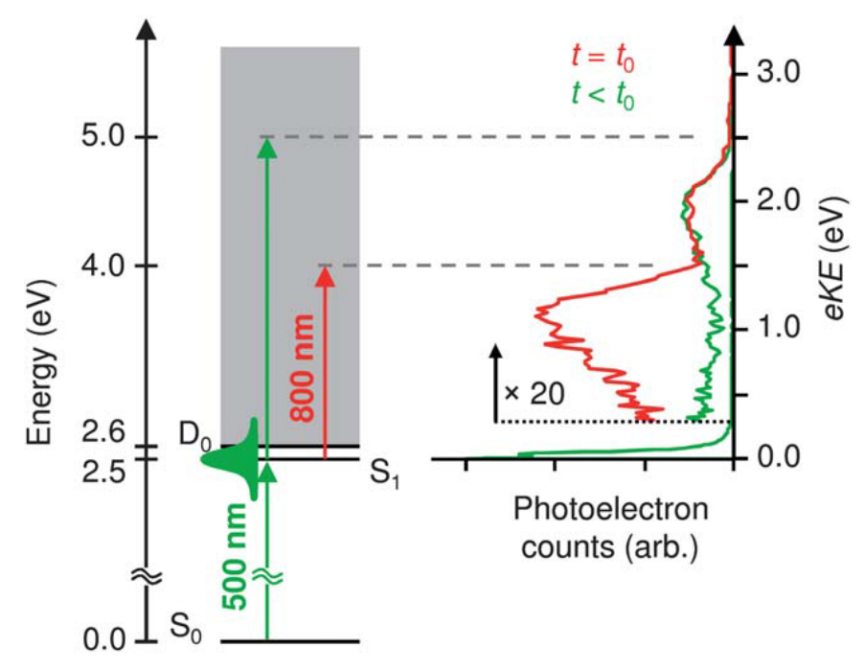

Figure 8. Left: Energy level diagram of $p$-HBDI ${ }^{-}$illustrating the pump-probe scheme employed to measure the relaxation dynamics following $500 \mathrm{~nm}(2.48 \mathrm{eV})$ photoexcitation of $\mathrm{S}_{1}$. Right: Photoelectron spectra recorded when the probe arrives before the pump $(t<0$, green) and with both pulses overlapped temporally $(t=0$, red). The large, non-dynamic feature at low eKE is attributed to indirect electron detachment from the anion. The pump-probe signature of $S_{1}$ is observed as a broad feature centred around $\mathrm{eKE}=1.1 \mathrm{eV}$. Reproduced from Ref. [22] with permission from the Royal Society of Chemistry.

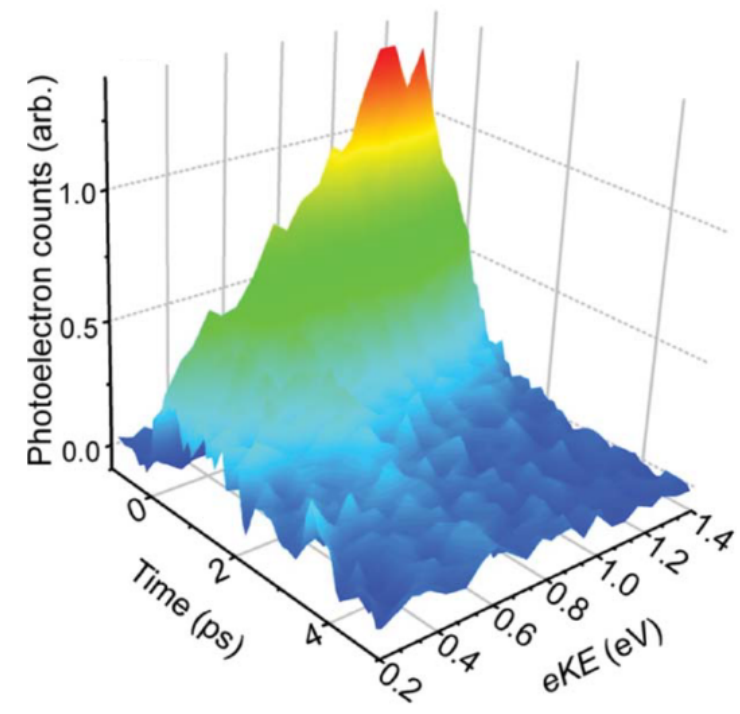

Figure 9. TRPES of $p$-HBDI ${ }^{-}$following photoexcitation of $\mathrm{S}_{1}$ at $500 \mathrm{~nm}(2.48 \mathrm{eV})$ and photodetachment at $800 \mathrm{~nm}(1.55 \mathrm{eV})$. Pump-only and probe-only spectra are subtracted from the pumpprobe spectra to leave only the time-resolved $\mathrm{S}_{1}$ excited state photoelectron signal as a function of time. The high eKE component is observed to decay on a sub-picosecond timescale leaving a low eKE component, centred around $0.6 \mathrm{eV}$, that decays more slowly. Reproduced from Ref. [22] with permission from the Royal Society of Chemistry. 
Supported by CASPT2/SA(2)-CASSCF $(16,14)$ calculations of the anion and corresponding neutral radical at key molecular geometries along the excited state reaction coordinate, it was proposed that the fast timescale $\left(\tau_{1}=330 \mathrm{fs}\right)$ was associated with a torsional motion in the C-C-C bridge and that the longer timescale $\left(\tau_{2}=1.4 \mathrm{ps}\right)$ was associated with subsequent IC from the $\mathrm{S}_{1}$ state back to $\mathrm{S}_{0}$. Since these timescales were in remarkable quantitative agreement with those measured following photoexcitation of the same chromophore in solution [76], it was concluded that the electronic relaxation mechanism is determined by the intrinsic electronic properties of the chromophore.

Subsequent time-resolved action-absorption measurements and detailed XMCQDPT2/SA(2)-CASSCF $(14,13)$ calculations of the potential energy landscape of gas-phase $p$-HBDI $^{-}$(Fig. 10) have revealed that the fast $\left(\tau_{1}=330 \mathrm{fs}\right.$ ) timescale can be attributed to twisting around the $\mathrm{C}-\mathrm{C}$ bond in the $\mathrm{C}-\mathrm{C}-\mathrm{C}$ bridge that is adjacent to the imidazolinone ring, $\theta[71]$. This work also showed that cooling gas-phase $p$-HBDI $^{-}$to $100 \mathrm{~K}$ traps population in a shallow well with a $0.05 \mathrm{eV}$ barrier on the excited state potential energy surface along the $\theta$ coordinate, for $1.2 \mathrm{~ns}$, which is long enough for fluorescence to occur.

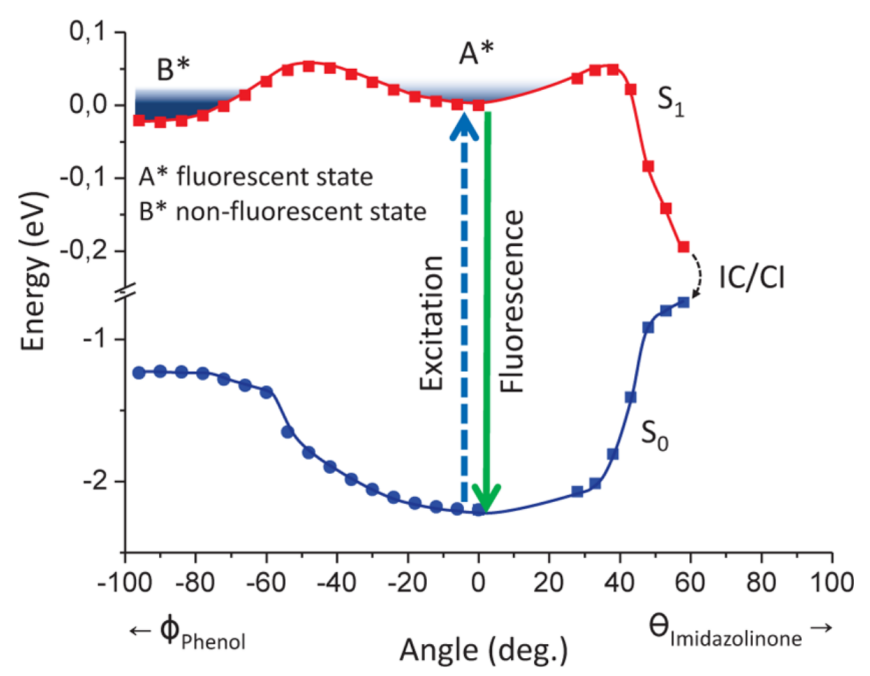

Figure 10. $\mathrm{S}_{0}$ and $\mathrm{S}_{1}$ potential energy curves as a function of $\mathrm{C}-\mathrm{C}$ twist angles in the C-C-C bridge adjacent to the imidazolinone and phenolate rings of $p$-HBDI ${ }^{-}$. IC/CI represents internal conversion through a conical intersection. Reproduced with permission from [71]. Copyright 2017 American Chemical Society.

\subsection{Higher lying electronically excited states}

In addition to their fluorescence properties, GFPs can also undergo irreversible phototransformation processes following irradiation with UV light [78]. One such example is decarboxylation (loss of $\mathrm{CO}_{2}$ ) of a glutamate residue (Glu222) that is believed to occur following photoexcitation of higher lying electronically excited states of the 
chromophore in its deprotonated anionic form [83]. Gas-phase photoelectron spectroscopy studies coupled with high-level quantum chemistry calculations have played an important role in characterising the dense manifold of higher lying electronic states that are involved in these UV transformation processes [19-21, 23, 26, 31]. The electron dynamics that govern the gas-phase UV photoelectron spectrum of $p$-HBDI ${ }^{-}$ have been found to be dominated by an optically bright, excited shape resonance, $\mathrm{S}_{3}$, which is strongly coupled to the $\mathrm{D}_{0}$ continuum. Scanning through the shape resonance with increasing photon energy has been shown to result in considerable broadening of the photoelectron spectrum that has been modelled by including both direct $\left(\mathrm{S}_{0}-\mathrm{D}_{0}\right)$ detachment and indirect $\left(\mathrm{S}_{3}-\mathrm{D}_{0}\right)$ detachment processes (Fig. 11).
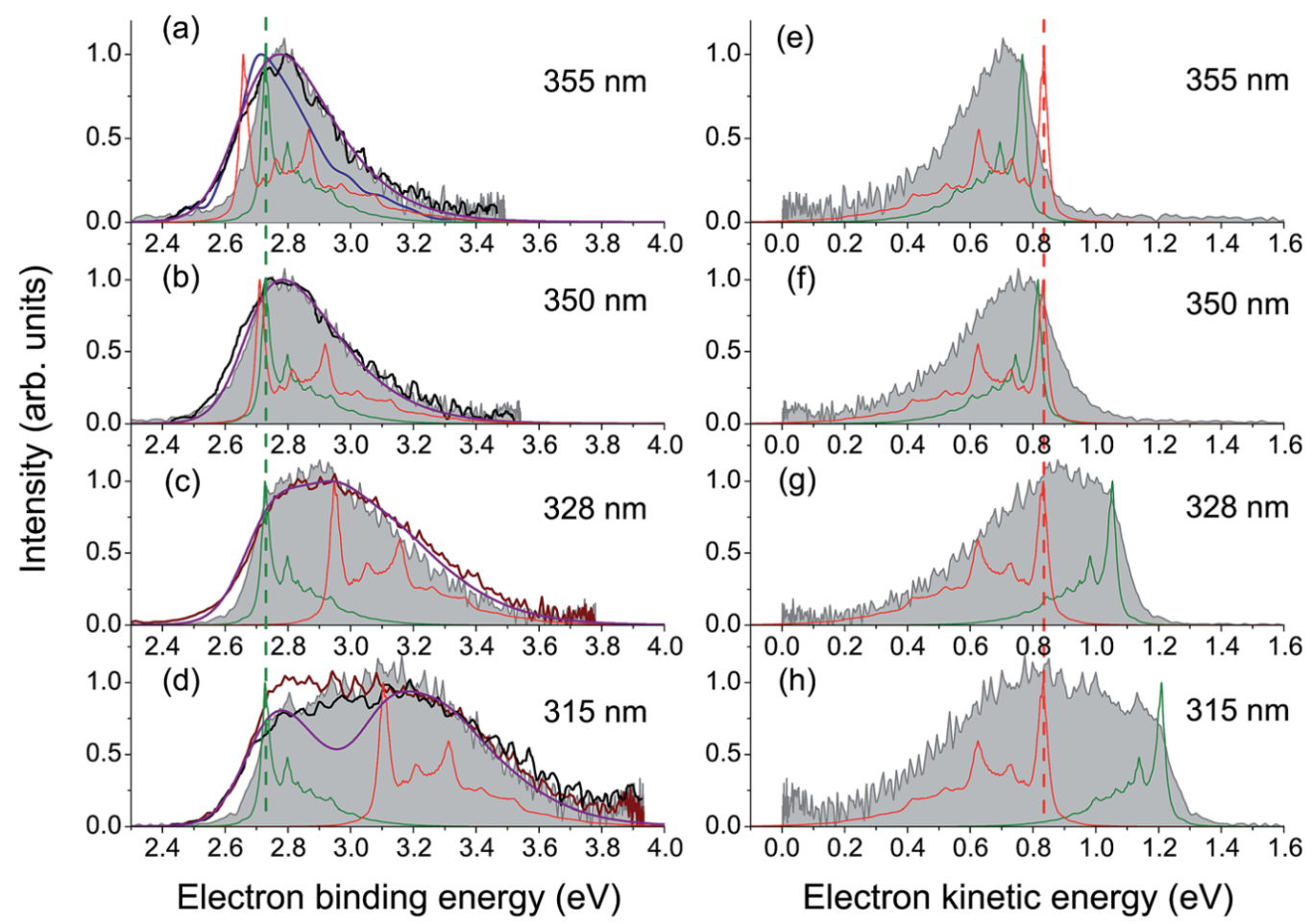

Figure 11. Experimental and calculated photoelectron spectra of $p$ HBDI $^{-}$following photoexcitation within the $\mathrm{S}_{0}-\mathrm{S}_{3}$ absorption band plotted as a function of eBE (left) and eKE (right). The experimental spectra are taken from a number of references: shaded grey [31]; brown (328 and $315 \mathrm{~nm})$ [23]; black $(355,350$ and $315 \mathrm{~nm})$ [26]; blue $(355 \mathrm{~nm})$ [21]. The calculated photoelectron spectra (purple) have contributions from direct $\left(\mathrm{S}_{0}-\mathrm{D}_{0}\right)$ detachment (green) and indirect $\left(\mathrm{S}_{3}-\mathrm{D}_{0}\right)$ detachment (red). Reproduced from Ref. [31] with permission from the Royal Society of Chemistry.

Understanding the pattern of excited states in the gas phase and their resonance character with respect to the detachment continuum is important for understanding the mechanism of resonant electron transfer from the deprotonated chromophore in the protein. Since the excited-shape resonance is strongly coupled to the detachment continuum in the gas phase, it is expected that resonant electron transfer out of this state in GFP should also be efficient. The calculated VEE of the excited 
shape resonance in the gas phase is $3.8 \mathrm{eV}(328 \mathrm{~nm})$. In the $\mathrm{S} 65 \mathrm{~T}$ GFP protein, this state is blue-shifted to $4.4 \mathrm{eV}(280 \mathrm{~nm})$ and is resonant with the continuum of a hydrated electron (Fig. 12) [31]. This state lies in the UV range in which oxidative decarboxylation of GFP has been observed, suggesting that the primary step in this photoconversion process may involve one-photon excitation in the UV or two-photon absorption in the visible range to the excited shape resonance followed by resonant electron transfer from the GFP chromophore anion to form the neutral radical.

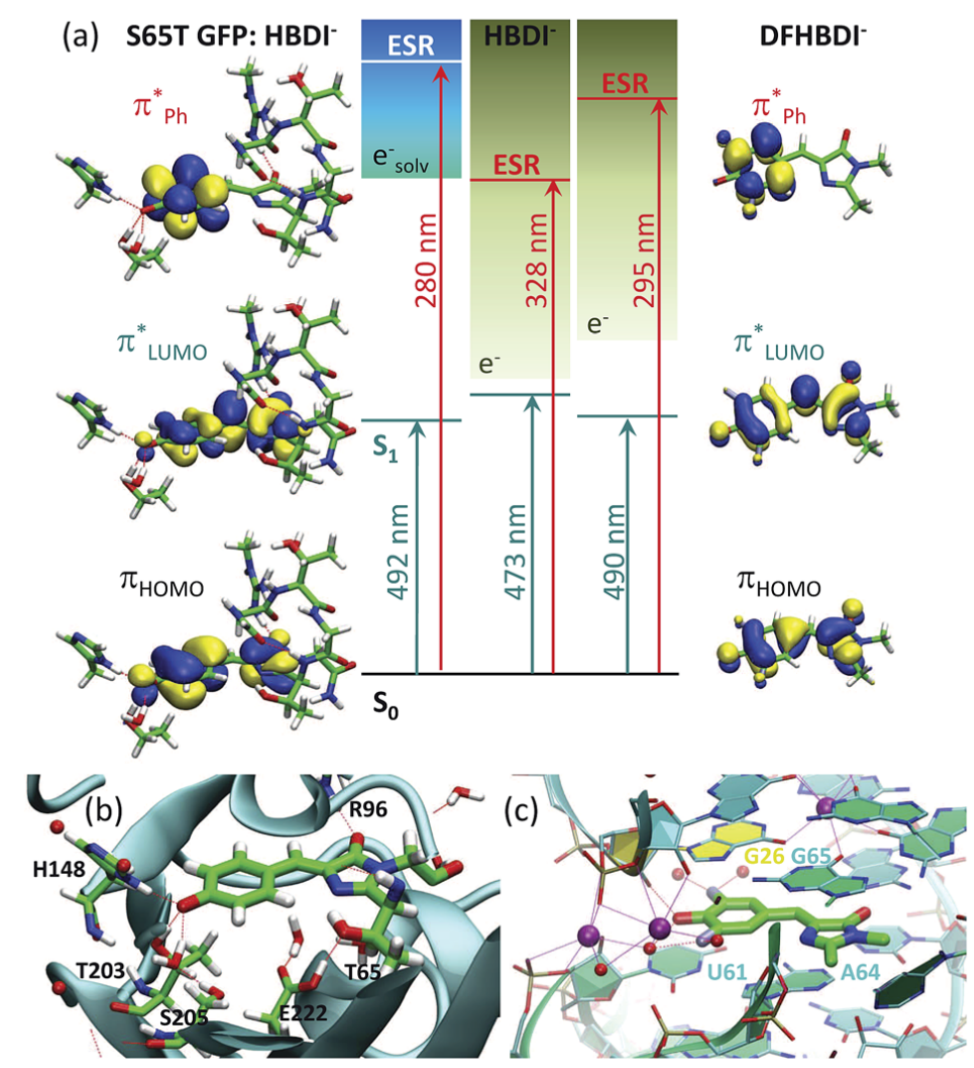

Figure 12. Electronic energy level diagram showing the $\mathrm{S}_{1}$ and $\mathrm{S}_{3}$ (excited shape resonance, ESR) VEEs and the VDEs for the following chromophores: the $p$-HBDI ${ }^{-}$chromophore in the gas phase (centre); the chromophore in the S65T GFP protein (left); the biomimetic chromophore, DFHBDI ${ }^{-}$, in the gas phase (right). The XMCQDPT2 natural orbitals involved in the $\mathrm{S}_{0}-\mathrm{S}_{1}\left(\pi_{\mathrm{LUMO}}^{*}-\pi_{\mathrm{HOMO}}\right)$ and $\mathrm{S}_{0}-\mathrm{S}_{3}\left(\pi_{\text {phenoxide }}^{*} \pi_{\text {HOMO }}\right)$ excitations are shown. Biological environments of $p$-HBDI ${ }^{-}$in S65T GFP (b) and of DFHBDI- in an RNA-fluorophore complex (c). Reproduced from Ref. [31] with permission from the Royal Society of Chemistry.

\subsection{Tuning electronic structure and relaxation dynamics}

Knowing that the protein environment of the chromophore plays a crucial role in enhancing and tuning the fluorescence properties of GFP has inspired several combined photoelectron spectroscopy and computational chemistry investigations of structurally modified chromophores aimed at understanding the role of the chromophore 
in tuning the electronic structure and relaxation dynamics; examples include substituting electron-donating and electron-withdrawing groups [31], moving the phenoxide group or adding an additional OH group to form a hydrogen-bonded 'lock' [33] and extending the conjugation [32] (Fig. 13).<smiles>COc1cc(/C=C2\N=C(C)N(C)C2=O)cc(OC)c1OC</smiles><smiles>COc1c(F)cc(/C=C2\N=C(C)N(C)C2=O)cc1F</smiles>

DFHBDI $^{-}$<smiles>CC1=N/C(=C\c2ccccc2[O-])C(=O)N1C</smiles><smiles>COc1ccc(/C=C2\N=C(C)N(C)C2=O)c(O)c1</smiles>

op-HBDI<smiles>CN1C(=O)/C(=C/C(=O)OC(C)(C)C)N=C1/C=C/c1cnc[n-]1</smiles>

Figure 13. Model deprotonated chromophores with electron-donating (DMHBDI $\left.{ }^{-}\right)$and electronwithdrawing $\left(\mathrm{DFHBDI}^{-}\right)$groups, with the phenoxide group moved to the ortho position $\left(o-\mathrm{HBDI}^{-}\right)$, with an additional $\mathrm{OH}$ group at the ortho position to form a hydrogen-bond 'lock' (op-HBDI ${ }^{-}$), and with extended conjugation (Kaede).

Deprotonated chromophores with electron-donating (DMHBDI ${ }^{-}$) and electronwithdrawing $\left(\mathrm{DFHBDI}^{-}\right)$groups have been shown to be fluorescent outside the native protein environment when bound to specific RNA sequences [84]. Using a combination of photoelectron spectroscopy and XMCQDPT2/aug-cc-pVTZ calculations, it has been shown that both difluoro and dimethoxy substituents blue shift the $\mathrm{S}_{3}$ excited-shape resonance (Section 3.4) by 0.3-0.4 eV [31], suggesting that these biomimetic chromophores are more stable to photooxidation. The significance of this is that higher energy photons will be required to initiate resonant electron transfer out of this state in the substituted chromophores, suggesting that the biomimetic GFP chromophores, $\mathrm{DMHBDI}^{-}$and $\mathrm{DFHBDI}^{-}$, are more protected against UV photooxidation in their biological RNA environment (Fig. 12).

In another combined photoelectron spectroscopy and quantum chemistry study, it was found that the low eKE (high eBE) component of the photoelectron spectrum was significantly enhanced in $o-\mathrm{HBDI}^{-}$compared to $p$-HBDI ${ }^{-}$and was significantly depleted in $o p-\mathrm{HBDI}^{-}$compared to $p$-HBDI ${ }^{-}$(Fig. 14) [33]. From these spectra and EOM-IP-CCSD/aug-cc-pVDZ, EPT/6-311++G(3df,3pd) and ADC(2)/aug-ccpVDZ calculations, it was concluded that moving the phenoxide group from the para position to the ortho position enhances internal conversion to lower lying electronic states but that adding an additional $\mathrm{OH}$ group to the para chromophore, at the ortho position, impedes internal conversion. These observations were interpreted in terms 
of the torsions around the $\mathrm{C}-\mathrm{C}-\mathrm{C}$ bridge being enhanced by electrostatic repulsions between the $\mathrm{O}^{-}$group in the ortho position and the $\mathrm{N}$ on the imidazolinone group, or impeded by the formation of a hydrogen-bond 'lock' between the $\mathrm{OH}$ group at the ortho position and the $\mathrm{N}$ on the imidazolinone group forming a seven-membered ring. It was also found that moving the phenoxide group from the para position to the ortho position reduced the energy required for detachment processes by around $0.2 \mathrm{eV}$, whereas adding an additional $\mathrm{OH}$ group to the para chromophore at the ortho position raised the energy required for detachment processes by around $0.2 \mathrm{eV}$.
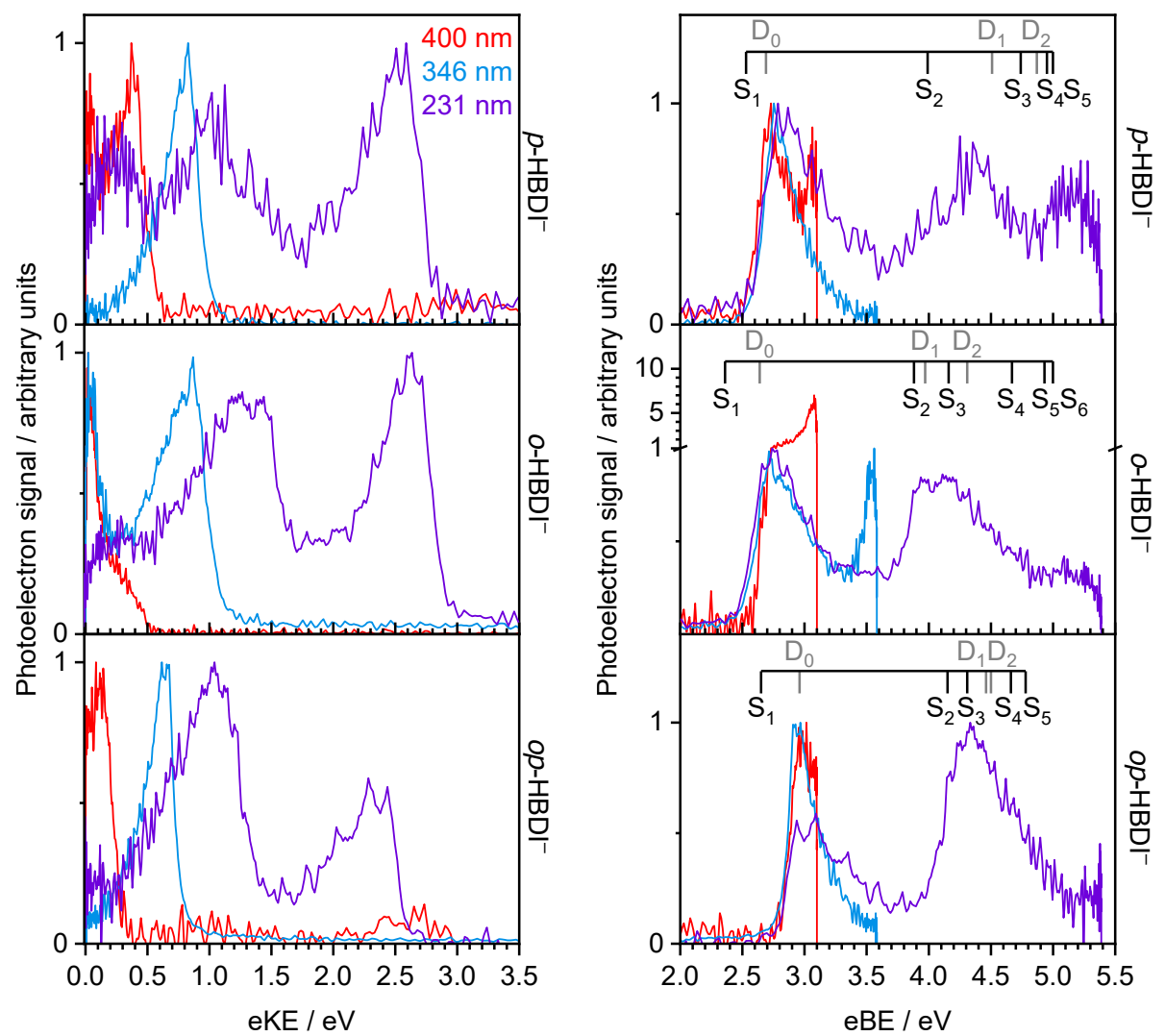

Figure 14. Photoelectron spectra of $p$-HBDI ${ }^{-}$(top), $o-\mathrm{HBDI}^{-}$(middle) and $o p-\mathrm{HBDI}^{-}$(bottom), recorded at $400 \mathrm{~nm}(3.10 \mathrm{eV}), 346.1 \mathrm{~nm}(3.582 \mathrm{eV})$ and $230.7 \mathrm{~nm}(5.374 \mathrm{eV})$ plotted as a function of eKE (left) and eBE (right). Intensities of the spectra plotted as a function of eKE (left) have been scaled to their maxima. Intensities of the spectra plotted as a function of eBE (right) have been scaled to align the rising edges of the low eBE features and are normalised to the maximum of their rising edges. Note the scale change used to plot the data for $o-\mathrm{HBDI}^{-}$. Combs mark the first three vertical detachment energies, calculated using the EOM-IP-CCSD/aug-cc-pVDZ method (grey), and the VEEs of the anions calculated using the ADC(2)/aug-cc-pVDZ method (black). Adapted from Ref. [33] with permission from the Royal Society of Chemistry.

Kaede, a green-to-red photoconvertible fluorescent protein that is an analogue of GFP, is used as an in vivo 'optical highlighter' in bioimaging. Curiously, it has a significantly lower fluorescence quantum yield $(\Phi \approx 0.33)$ than GFP [85]. Using a combination of photoelectron spectroscopy measurements of the deprotonated red Kaede chromophore in the gas phase and MS-CASPT2(14,14)/ANO-L calculations, 
it was found that there is an efficient electronic relaxation pathway from higher lying electronically excited states, which have Feshbach resonance character with respect to the detachment continuum, to the $\mathrm{S}_{1}$ state, which has shape resonance character, that is not in operation in $p$-HBDI ${ }^{-}$[32]. Unlike the photoelectron spectra of $p$ $\mathrm{HBDI}^{-}$, the photoelectron spectra of the red Kaede chromophore were found to have significant intensities at low eKE (high eBE), but they did not have the characteristic shape associated with thermionic emission from the ground electronic state (Fig. 15). Thus, it was concluded that following relaxation from higher lying excited electronic states to $\mathrm{S}_{1}$, vibrational autodetachment was an efficient relaxation pathway in the red Kaede chromophore. If the $\mathrm{S}_{1}$ state in the protein is resonant with the continuum of a hydrated electron, this could explain why the fluorescence quantum yield of red Kaede is lower than that of GFP; however, it should be noted that the isolated red Kaede protein chromophore in the gas phase is deprotonated at the imidazole ring (Fig. 13), unlike the situation in the protein where it is deprotonated at the phenol ring.

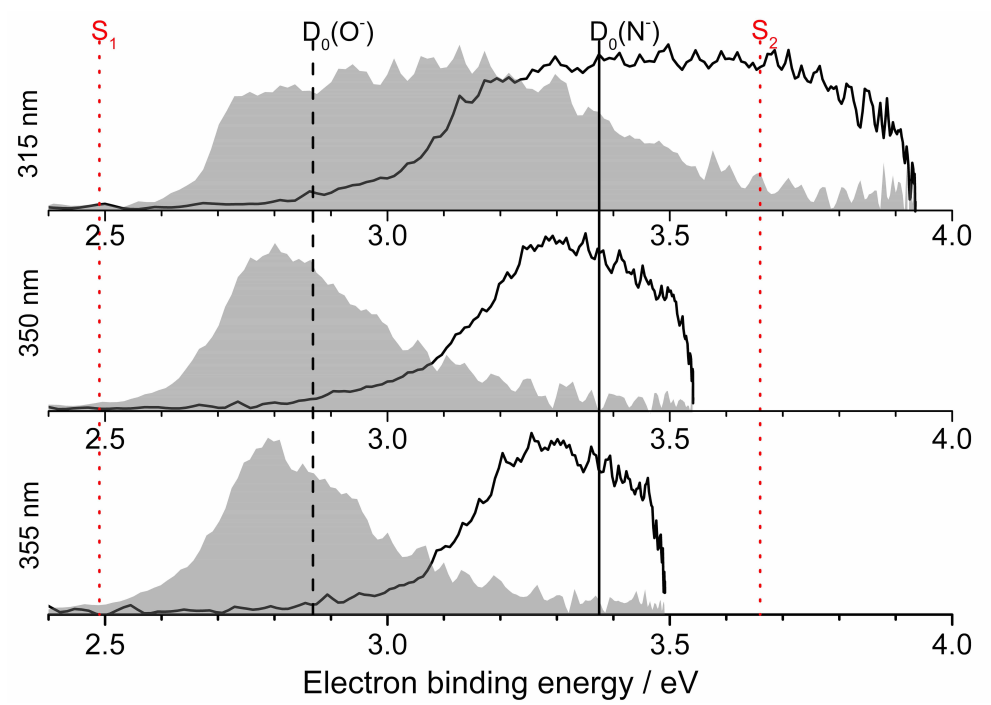

Figure 15. Photoelectron spectra of the red Kaede chromophore recorded at $355 \mathrm{~nm}(3.49 \mathrm{eV}), 350$ $\mathrm{nm}(3.54 \mathrm{eV})$, and $315 \mathrm{~nm}(3.94 \mathrm{eV})$ (solid black lines) compared with photoelectron spectra of $p$-HBDI ${ }^{-}$(shaded gray). Black vertical lines mark the EPT/6-311++G(3dp,3df) calculated VDEs of the red Kaede chromophore deprotonated at the $\mathrm{N}$ atom (solid) and $\mathrm{O}$ atom (dashed), and dotted red vertical lines mark the MS-CASPT2 $(14,14) /$ ANO-L VEEs of the first two excited singlet states of the deprotonated Kaede chromophore. Reprinted with permission from [32]. Copyright (2017) American Chemical Society.

An alternative approach to tuning the electronic structure of the chromophore is to modify the microenvironment of the chromophore. A high resolution vibrationallyresolved photoelectron spectroscopy study of microsolvated GFP chromophores, $p$ $\mathrm{HBDI}^{-}\left(\mathrm{H}_{2} \mathrm{O}\right)_{n}$ with $n=0,1,2$, has shown that individual solvent water molecules have a significant stabilisation effect on the deprotonated chromophore anion relative to the detachment continuum [27]. The VDE has been found to increase from $2.73 \mathrm{eV}$ 
for the bare anion to $3.15 \mathrm{eV}$ when it was solvated with a single water molecule and $3.50 \mathrm{eV}$ with two water molecules. Supporting EOM-IP-CCSD/maug-cc-pVTZ calculations showed that the first two water molecules are bound to the phenoxide moiety of the chromophore. This work is particularly significant as it demonstrates how just one or two water molecules, like crystallographic water molecules, can have a dramatic impact on the electronic structure of GFP chromophores.

\section{Photoactive yellow protein chromophores}

\subsection{Background}

Photoactive yellow protein (PYP) is the primary photoreceptor responsible for the negative phototactic response of the Halorhodospira halophila bacterium upon absorption of blue light, limiting its exposure to potentially harmful UV light [5]. The chromophore that lies at the heart of PYP is trans-para-coumaric acid $(p \mathrm{CA})$; the deprotonated anionic form, $p \mathrm{CA}^{-}$, is shown in Fig. 16.<smiles>COc1ccc(/C=C/C(=O)O)cc1</smiles>

Figure 16. Structure of the deprotonated PYP chromophore anion, $p \mathrm{CA}^{-}$.

In PYP, the chromophore is covalently bound to the protein by a thio-ester link with a cysteine residue. The negative charge on the chromophore is stabilised by a network of hydrogen bonds to surrounding amino acid residues. Following photoexcitation, the deprotonated chromophore anion undergoes rapid trans-cis isomerisation to generate a red-shifted intermediate, $\mathrm{pR}$, in which the chromophore is in a strained cis conformation (Fig. 17). The hydrogen-bond network then reorganises to accommodate the cis conformation and the protein partially unfolds and the chromophore is protonated, generating a blue-shifted intermediate, $\mathrm{pB}$, which is the signalling state. Subsequent cis-trans isomerisation of the chromophore, refolding of the protein and deprotonation of the chromophore, completes the photocycle by regenerating the protein in its initial state, $\mathrm{pG}$.

The initial photoisomerisation process in PYP is similar to that in rhodopsin, one of nature's most important photoreceptors [86]. PYP is an ideal system for studying biological photoreceptors as a result of its small size (125 residues), stability and ease of crystallisation [86-90]. Numerous spectroscopic and computational chemistry studies of the electronic structure and relaxation dynamics of isolated model PYP chromophores in the gas phase have been aimed at understanding the role of the chromophore and its environment in determining the function of this important photoreceptor [25, 28, 29, 34, 59, 91-95]. 


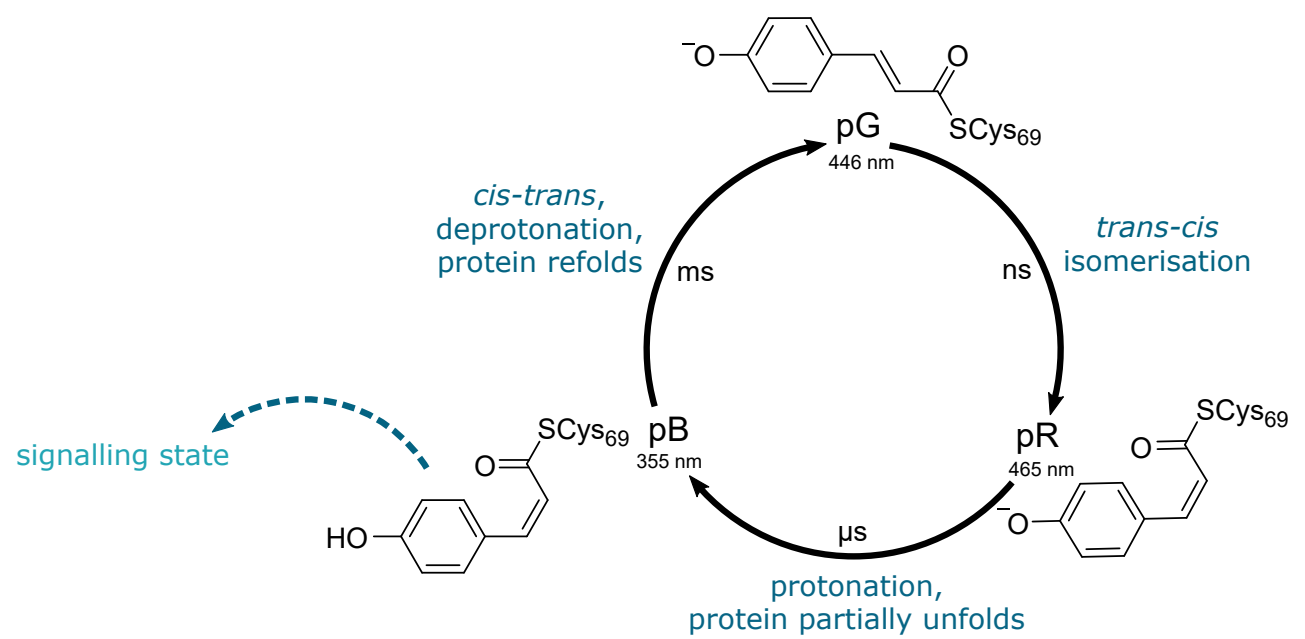

Figure 17. Photocycle of photoactive yellow protein (PYP) showing the major intermediates, their absorption maxima and time-scales.

\subsection{Vertical detachment energy}

The first experimental study of the electronic structure of gas-phase $p \mathrm{CA}^{-}$was an action-absorption spectroscopy study [91]. The action-absorption band was found to have a maximum around $430 \mathrm{~nm}(2.88 \mathrm{eV})$, close to the absorption maximum of the protein $(446 \mathrm{~nm}$ ). This led to the suggestion that, as for GFP (Section 3.2), the electrostatic environment of the PYP chromophore in the protein is closer to that of the chromophore in vacuo than the chromophore in aqueous solution. There has been some discussion about the deprotonated form of the $p \mathrm{CA}$ chromophore in vacuo $[59,91,93,95]$ as it has acidic hydrogen sites on the phenol and carboxylic acid groups and can therefore exist as phenolate and carboxylate anions (Fig. 18), and experimental conditions have been shown to play an important role in determining the deprotonation site during ESI experiments [95]. In the gas phase, the phenolate is more stable than the carboxylate because the negative charge is delocalised throughout the anion, but in polar solvents, the carboxylate is more stable than the phenolate because the more localised charge can be solvated more efficiently. SA-CAS(14,12)-PT2/cc-pVDZ calculations have predicted VEEs of $2.89 \mathrm{eV}$ and 3.86 $\mathrm{eV}$ for the phenolate and carboxylate forms, respectively [96], suggesting that the action-absorption spectra were dominated by the phenolate form of $p \mathrm{CA}^{-}$.<smiles>COc1ccc(/C=C/C(=O)O)cc1</smiles><smiles>O=C([O-])/C=C/c1ccc(O)cc1</smiles>

Figure 18. Phenolate and carboxylate deprotonated forms of $p \mathrm{CA}^{-}$. 
The photodetachment process in gas-phase $p \mathrm{CA}^{-}$has been measured in a number of photoelectron spectroscopy studies $[25,28,29]$ and the most accurate value for the VDE obtained experimentally is $2.92 \pm 0.05 \mathrm{eV}$ [29]. The VDEs of the two isomers of $p \mathrm{CA}^{-}$calculated using the EPT/6-311++G(3df,3pd) method were found to be $3.00 \mathrm{eV}$ for the phenolate form and $4.68 \mathrm{eV}$ for the carboxylate form, suggesting that the photoelectron spectra were dominated by the phenolate form of $p \mathrm{CA}^{-}$[25].

\section{3. $\mathrm{S}_{1}$ dynamics}

The timescales associated with electronic relaxation following photoexcitation of the $\mathrm{S}_{1}$ state of a model keto PYP chromophore, $p \mathrm{CK}^{-}$(Fig. 19), in vacuo have been determined using TRPES [92]. Gas-phase anions were excited with $400 \mathrm{~nm}(3.10 \mathrm{eV})$ femtosecond pump pulses, close to the absorption maximum. The evolution of the population on the excited state was monitored by firing $800 \mathrm{~nm}(1.55 \mathrm{eV})$ femtosecond probe pulses at a series of pump-probe delays to project the excited state population onto the photodetachment continuum.<smiles>COc1ccc(/C=C/C(C)=O)cc1</smiles>

Figure 19. Structure of the model keto PYP chromophore, $p \mathrm{CK}^{-}$, used in TRPES experiments [92].

The pump-only spectrum was observed to have an intense peak around $0.2 \mathrm{eV} \mathrm{eKE}$, arising from one-photon direct photodetachment from the ground state (Fig. 20). In the pump-probe spectrum at $200 \mathrm{fs}$ delay, the peak around $0.2 \mathrm{eV}$ was depleted and a broad peak centred around $0.9 \mathrm{eV}$ appeared. With increasing pump-probe delay, the low energy feature around $0.2 \mathrm{eV}$ (labelled I in Fig. 20) was observed to be recovered on timescales of $4 \mathrm{ps}$ and $52 \mathrm{ps}$. The broad peak around $0.9 \mathrm{eV}$ was separated into two regions (labelled II and III in Fig. 20). The higher eKE region II was observed to decay on timescales of 1 ps and 4 ps. The lower eKE region III was observed to rise on a timescale of $1 \mathrm{ps}$ and then decay on a timescale of $52 \mathrm{ps}$.

These TRPES spectra showed that following photoexcitation, population on the excited $\mathrm{S}_{1}$ state initially bifurcated with $80 \%$ of the population remaining on the excited state of the chromophore in its trans form, $\mathrm{P}_{t}^{*}$ (region II), and $20 \%$ of the population undergoing autodetachment to form a low eKE electron and neutral radical, $\mathrm{P}_{e}^{*}$ (region I), on a 4 ps timescale. $\mathrm{P}_{t}^{*}$ then evolved to form a twisted intermediate, $\mathrm{P}_{\theta}^{*}$ (region III), on a 1 ps timescale, which then underwent internal conversion, on a 52 ps timescale, back to the electronic ground state as a vibrationally hot cis conformer, $\mathrm{P}_{c}$. The decay of $\mathrm{P}_{\theta}^{*}$ was accompanied by a damped oscillation with period corresponding to $\sim 40 \mathrm{~cm}^{-1}$, a low-frequency motion involved in the isomerisation.

The timescale for trans-cis isomerisation of this particular chromophore in the gas phase (52 ps) is an order of magnitude slower than isomerisation in the protein; this was explained in terms of IVR through coordinates orthogonal to the reaction path 


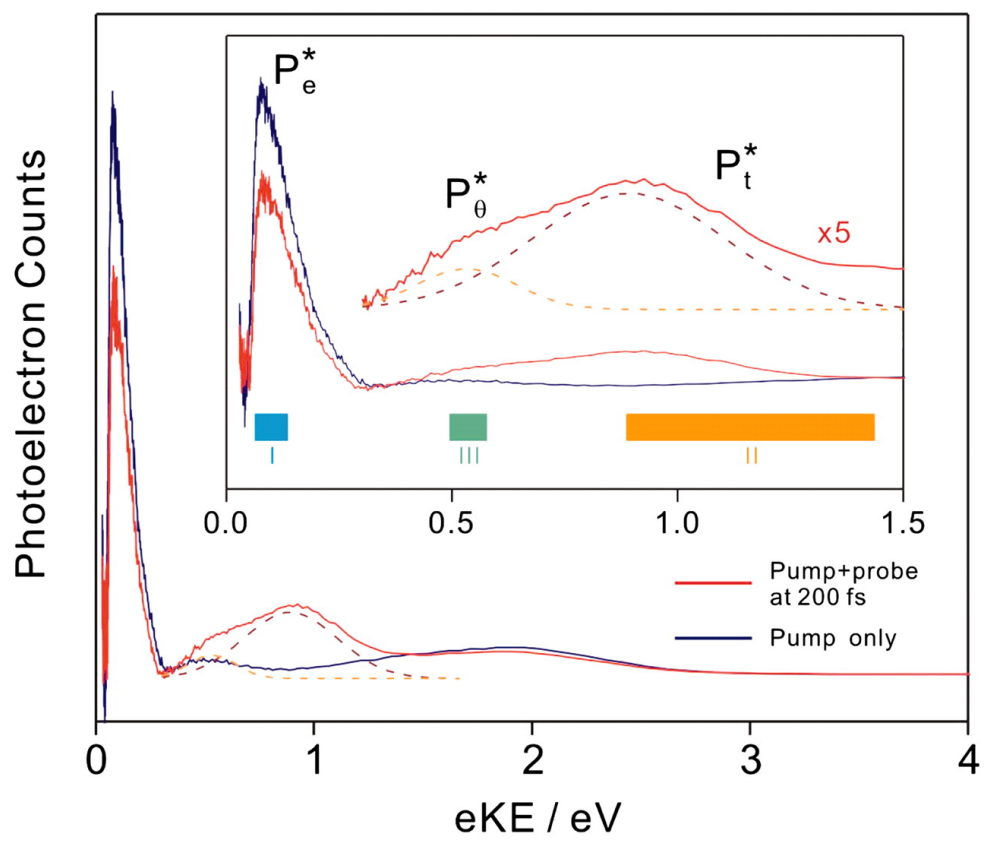

Figure 20. Pump-only (blue) and pump-probe (200 fs delay; red) photoelectron spectra of $p \mathrm{CK}^{-}$. Inset: $0-1.5 \mathrm{eV}$ eKE region with eKE windows I, II and III, corresponding to $\mathrm{P}_{e}^{*}, \mathrm{P}_{t}^{*}$ and $\mathrm{P}_{\theta}^{*}$, respectively (see text). Reproduced from Ref. [92], copyright 2006 National Academy of Sciences.

(Fig. 21). It was concluded that one of the roles of the microenvironment within the PYP protein is therefore to direct excited state population towards the conical intersection with the cis ground-state with high quantum yield $(\Phi \sim 0.35$ [97-101]). This also supported earlier QM/MM calculations predicting that the conical intersection between $\mathrm{S}_{1}$ and the cis ground state lay further from the global minimum on the $\mathrm{S}_{1}$ potential energy surface for the chromophore in vacuo than in the protein (Fig. 21 inset) [102].

\subsection{Higher lying electronically excited states}

Gas-phase photoelectron studies coupled with quantum chemistry calculations have played an important role in characterising the dense manifold of higher lying electronic states. Higher lying excited states of PYP chromophores have been implicated in the formation of solvated electrons from isolated chromophores in solution $[103,104]$ and from the protein [105-107], suggesting that the protein pocket provides a similar environment for a solvated electron as bulk water.

Anion photoelectron spectra of $p \mathrm{CA}^{-}$recorded at photon energies in the range 330-310 nm have been shown to be broadened considerably and this broadening was attributed to autodetachment from a resonance in the continuum (Fig. 22). SACASSCF $(14,12)-P T 2$ calculations found the VEEs of the first three singlet electronically excited states to be $419 \mathrm{~nm}(2.96 \mathrm{eV}), 339 \mathrm{~nm}(3.65 \mathrm{eV})$ and $325 \mathrm{~nm}(3.82 \mathrm{eV})$ [57], in agreement with action-absorption measurements [93]; these transitions were dominated by excitations of $\mathrm{S}_{1}\left(\pi \pi^{*}\right), \mathrm{S}_{2}\left(n \pi^{*}\right)$ and $\mathrm{S}_{3}\left(\pi \pi^{*}\right)$ character, respectively. 


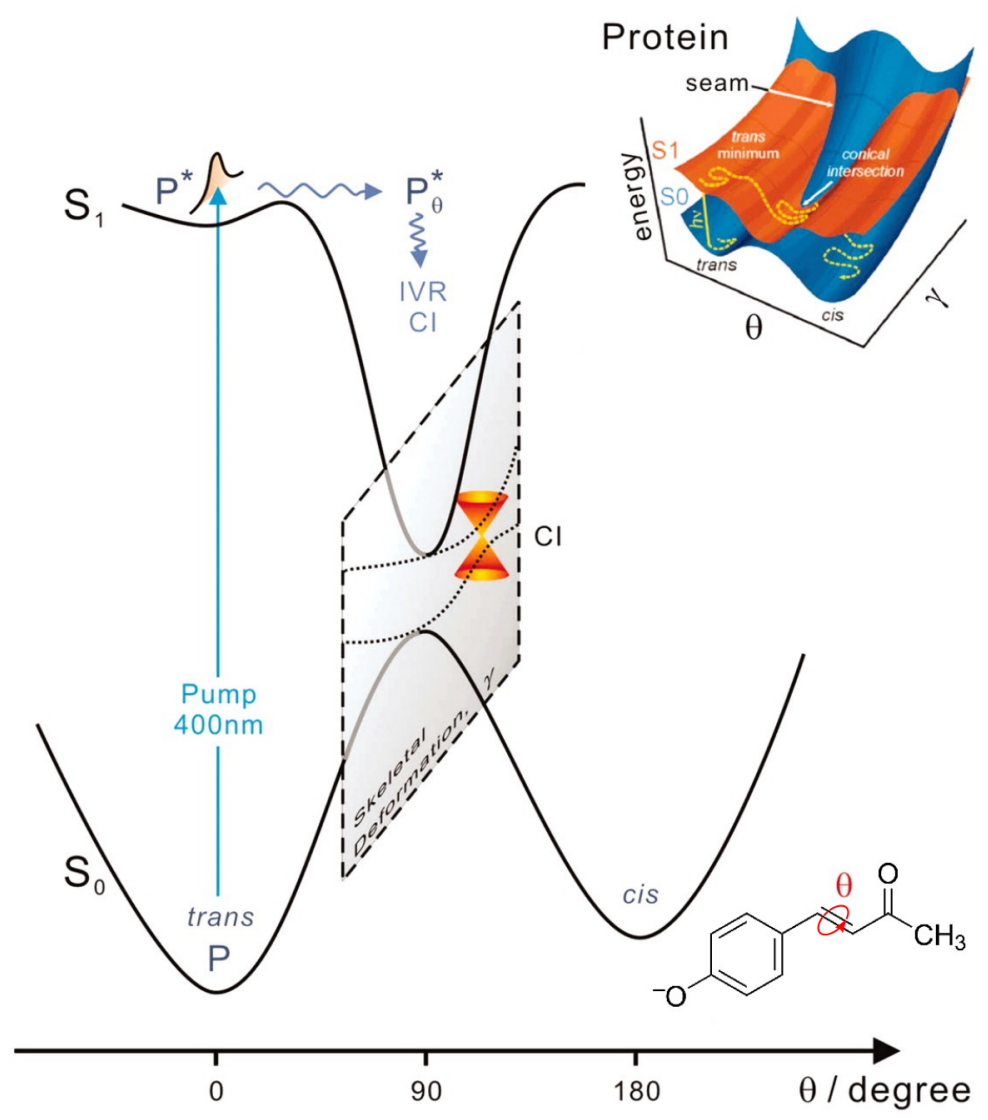

Figure 21. Schematic potential energy surface illustrating photoisomerisation of $p \mathrm{CK}^{-}$. The photoisomerisation coordinate is $\theta$ and orthogonal coordinates are $\gamma$, represented by the perpendicular plane labelled skeletal deformation and containing the conical intersection (CI) with the cis groundstate. Following photoexcitation from $\mathrm{S}_{0}$ to $\mathrm{S}_{1}$, population on the excited state $\left(\mathrm{P}^{*}\right)$ bifurcates to $\mathrm{P}_{e}^{*}(20 \%)$ and $\mathrm{P}_{t}^{*}(80 \%)$. Inset: illustration of the dynamics of the chromophore in PYP with a cut in the $3 \mathrm{D}$ surface showing a similar $\theta$ dependence but having a CI much closer to the reaction coordinate. Reproduced from Ref. [92], copyright 2006 National Academy of Sciences.

The oscillator strength of $\mathrm{S}_{1}$ was near unity, while that of $\mathrm{S}_{2}$ was zero (a dark state) and that of $S_{3} \sim 10 \%$. The first two singlet excited states lying above the detachment threshold, $\mathrm{S}_{2}$ and $\mathrm{S}_{3}$, have Feshbach and excited-shape resonance character with respect to the $\mathrm{D}_{0}$ continuum, respectively, suggesting that the broadening of photoelectron spectra following excitation around $320 \mathrm{~nm}$ arises from fast autodetachment from the $\mathrm{S}_{3}$ excited-shape resonance.

Since the excited-shape resonance is strongly coupled to the detachment continuum in the gas phase, it is expected that resonant electron transfer from this state of the chromophore in aqueous solution, or in PYP, would also be efficient and could be responsible for the generation of solvated electrons. In the gas phase, a feature with $\mathrm{eKE} \sim 0$ has been attributed to thermionic emission from $\mathrm{S}_{0}$ (Fig. 22), suggesting that internal conversion from $\mathrm{S}_{3}$ to the ground state competes with autodetachment. 

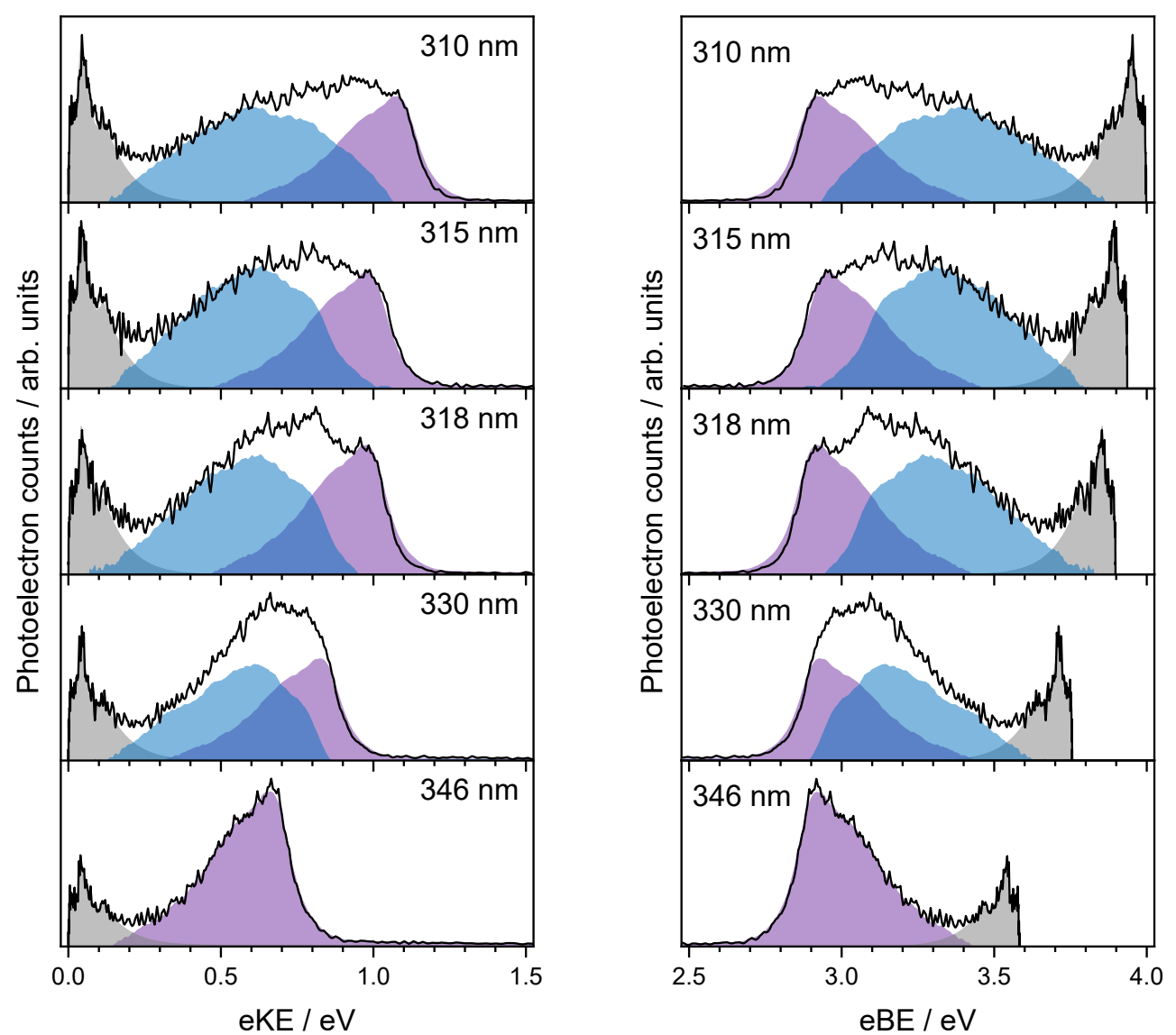

Figure 22. Photoelectron spectra of $p \mathrm{CA}^{-}$at wavelengths of $310 \mathrm{~nm}(4.00 \mathrm{eV}), 315 \mathrm{~nm}(3.94 \mathrm{eV})$, $318 \mathrm{~nm}(3.90 \mathrm{eV}), 330 \mathrm{~nm}(3.76 \mathrm{eV})$ and $346 \mathrm{~nm}(3.58 \mathrm{eV})$, plotted as a function of eKE and eBE. Direct photodetachment from $\mathrm{S}_{0}$ to $\mathrm{D}_{0}$ gives rise to photoelectrons with a maximum eKE that corresponds to the $\mathrm{S}_{0}-\mathrm{D}_{0} \mathrm{VDE}$ (purple). TE from $\mathrm{S}_{0}$ following photoexcitation of an electronically excited state of the anion and subsequent IC back to $\mathrm{S}_{0}$ gives electrons with eKE $\sim 0$ (grey). Spectral profiles corresponding to direct detachment and TE processes are determined from the $346 \mathrm{~nm}$ spectrum. The spectral profiles corresponding to indirect photodetachment (blue) are obtained by subtracting direct detachment and TE spectral profiles from each photoelectron spectrum; the maxima of the indirect photodetachment spectral profiles correspond, approximately, to $E\left(\mathrm{~S}_{n}\right)$ $E\left(\mathrm{D}_{0}\right) \sim 0.6 \mathrm{eV}$ eKE. From the experimentally determined VDE $(\sim \mathrm{ADE})=2.92 \mathrm{eV}[29], E\left(\mathrm{~S}_{n}\right) \sim$ $3.5 \mathrm{eV}$, in agreement with the onset (adiabatic excitation energy) of the second band in the actionabsorption spectrum of $p \mathrm{CE}^{-}$[93], a related chromophore. Data from Ref. [29] with permission from the PCCP Owner Societies.

\subsection{Tuning electronic structure and relaxation dynamics}

Knowing that the structure of the chromophore and the protein environment play an important role in tuning the electronic relaxation dynamics has inspired several combined anion photoelectron spectroscopy and quantum chemistry investigations of structurally modified chromophores; examples include changing the position of the phenoxide group ( $o \mathrm{CA}^{-}$and $m \mathrm{CA}^{-}$) [25], substitution at the coumaryl tail $\left(p \mathrm{CT}^{-}\right.$ and $\left.p \mathrm{CE}^{-}\right)$[28] or introducing single-bond $\left(\mathrm{P} p \mathrm{CE}^{-}\right.$and $\left.\mathrm{N} p \mathrm{CE}^{-}\right)$[29] or double-bond (EBp $\left.\mathrm{CE}^{-}\right)$[34] 'locks' (Fig. 23).

A study of $o \mathrm{CA}^{-}, m \mathrm{CA}^{-}$and $p \mathrm{CA}^{-}$chromophores showed that moving the posi- 
<smiles>O=C(O)/C=C/c1ccccc1[O-]</smiles><smiles>COc1cccc(/C=C/C(=O)O)c1</smiles><smiles>COc1ccc(/C=C/C(=O)SC)cc1</smiles><smiles>COC(=O)/C=C/c1ccc(OC)cc1</smiles><smiles>COc1ccc(/C=C2\CCCOC2=O)cc1</smiles><smiles>COC(=O)/C=C1\CCCc2cc(OC)ccc21</smiles><smiles>COc1ccc(C2=CC(=C(C)C)OCC2)cc1</smiles>

Figure 23. Model deprotonated chromophores with the phenoxide group moved to ortho $\left(o \mathrm{CA}^{-}\right)$ and meta $\left(m \mathrm{CA}^{-}\right)$positions, substitution of ester $\left(p \mathrm{CE}^{-}\right)$and thioester $\left(p \mathrm{CT}^{-}\right)$groups at the coumaryl tail, and 'locked' single- $\left(\mathrm{P} p \mathrm{CE}^{-}, \mathrm{N} p \mathrm{CE}^{-}\right)$and double- $\left(\mathrm{EB} p \mathrm{CE}^{-}\right)$bonds.

tion of the $\mathrm{O}^{-}$group on the phenoxide moiety changed the branching ratio between direct photodetachment and indirect photodetachment. In agreement with action absorption spectra [93], CAM-B3LYP/6-311++G(3df,3pd) calculated VEEs showed that the $\mathrm{S}_{3}$ state was much higher in energy for the ortho isomer than the other isomers and that the $\mathrm{S}_{1}$ state was significantly lower and had almost negligible oscillator strength for the meta isomer. Thus, following photoexcitation in the range 364-315 nm, the $\mathrm{S}_{3}$ state of $o \mathrm{CA}^{-}$was inaccessible so the spectra did not show the broadening characteristic of indirect detachment observed in $m \mathrm{CA}^{-}$and $p \mathrm{CA}^{-}$. Moreover, the longer wavelength photoelectron spectra of $\mathrm{mCA}^{-}$were found to be completely dominated by direct photodetachment.

A separate study of $p \mathrm{CA}^{-}, p \mathrm{CE}^{-}$and $p \mathrm{CT}^{-}$was aimed at investigating how substitution at the coumaryl tail of the chromophore controls competing relaxation pathways following UV photoexcitation in the range 350-315 $\mathrm{nm}$ [28]. The photoelectron spectra were found to be dominated by electrons arising from direct detachment and indirect detachment from the $\mathrm{S}_{3}\left(\pi \pi^{*}\right)$ state, but some spectra also had a low eKE component arising from autodetachment from lower lying electronically excited states or thermionic emission from the electronic ground state (Fig. 24). Substituting the hydrogen atom of the carboxylic acid group $\left(p \mathrm{CA}^{-}\right)$with a methyl group $\left(p \mathrm{CE}^{-}\right)$was found to lower the threshold for electron detachment but had very little effect on the competition between the different relaxation pathways. However, substituting with a thioester group $\left(p \mathrm{CT}^{-}\right)$raised the threshold for electron detachment and appeared to 'turn off' the competing electron emission processes from lower lying electronically excited states. This has potential implications in terms of tuning the light-induced electron donor properties of PYP.

Computational studies of $\mathrm{S}_{1}$ dynamics have found a minimum on the $\mathrm{S}_{1}$ potential energy surface involving a twist around the single bond connecting the phenolate and double bond moeities, in addition to the minimum along the isomerisation coordinate $[102,108-112]$. This led to the suggestion that single-bond rotation may compete with isomerisation (double-bond rotation) and therefore impact the relaxation path- 

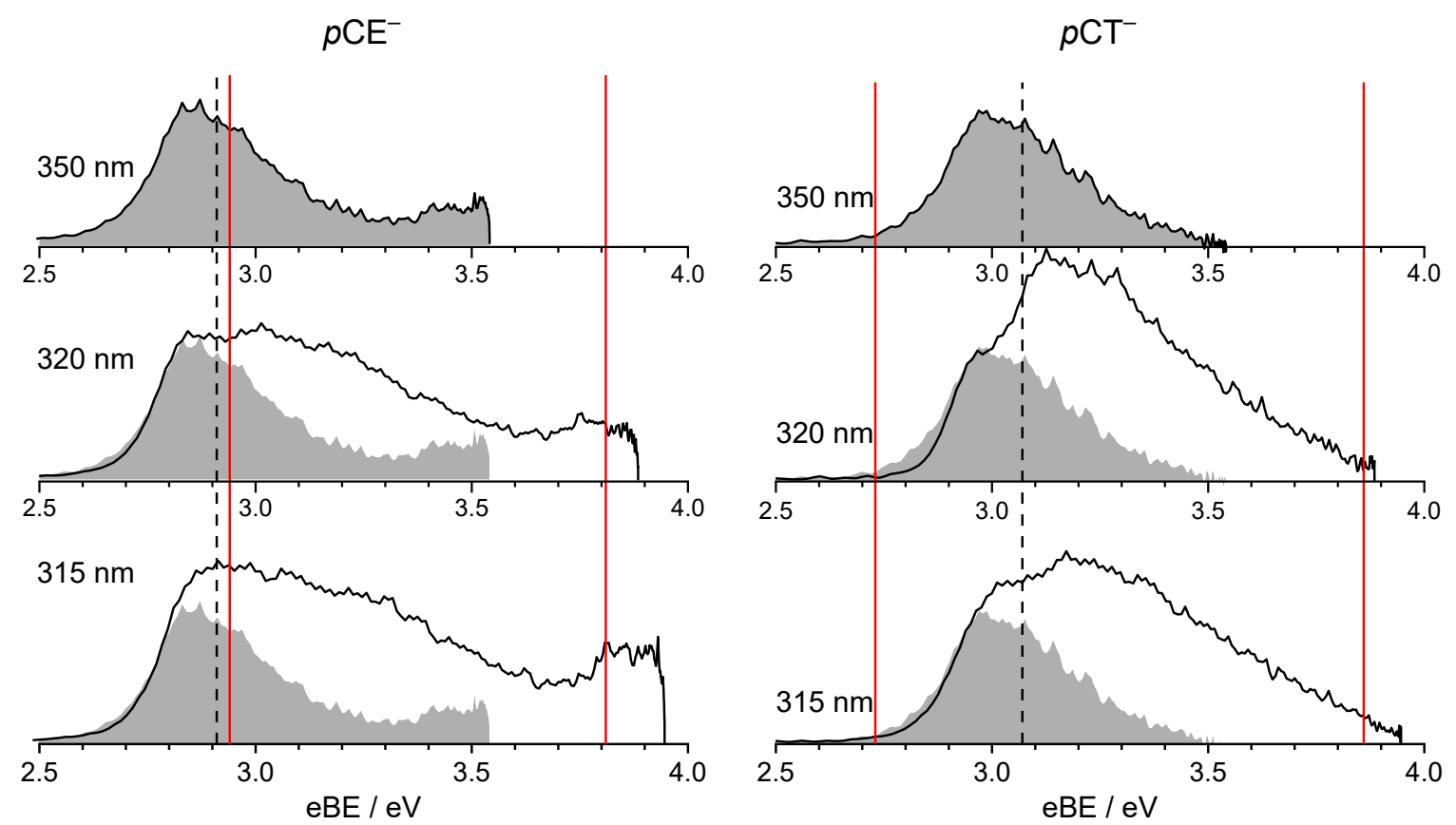

Figure 24. Photoelectron spectra of $p \mathrm{CE}^{-}$and $p \mathrm{CT}^{-}$recorded at $350 \mathrm{~nm}(3.54 \mathrm{eV}), 320 \mathrm{~nm}$ $(3.87 \mathrm{eV})$ and $315 \mathrm{~nm}(3.94 \mathrm{eV})$ presented as a function of electron binding energy (eBE). The $350 \mathrm{~nm}$ spectra are superimposed on the 310 and $315 \mathrm{~nm}$ spectra, as shown in shaded grey. Intensities have been scaled to align the rising edges. Dashed black lines mark the calculated VDEs (EPT/6-311++G(3df,3pd)) and solid red lines mark the VEEs of the $S_{1}$ and $S_{3}$ states calculated using SA-CASSCF(14,12)-PT2/cc-pVDZ by Garcia-Prieto et al. [57]. Reproduced from Ref. [28] with permission from the PCCP Owner Societies.

way following photoexcitation of the chromophore in the gas phase. Recent anion photoelectron spectroscopy measurements of 'locked' chromophores combined with $\mathrm{ADC}(2) / 6-31+\mathrm{G}^{* *}$ calculations have led to the suggestion that locking the double bond turns off internal conversion to the ground state and enhances electron emission from $\mathrm{S}_{1}$ [34]. Indirect electron emission from $\mathrm{S}_{1}$ was distinguished from thermionic emission by fitting Eq. (3) to the photoelectron distribution (Fig. 25). It has also been shown that locking the single bonds either side of the double bond reduces the efficiency of $\mathrm{S}_{1}-\mathrm{S}_{0}$ internal conversion and that this effect is more pronounced for the single bond connecting the double bond with the phenolate moiety [29] (Fig. 26).

\section{Luciferins}

\subsection{Background}

The brightest bioluminescence found in nature is that of the Photinus pyralis firefly which has a quantum yield, $\Phi=0.41$ [6]. In fireflies, a luciferase enzyme catalyses the adenylation of deprotonated luciferin (Fig. 27) by adenosine triphosphate (ATP) to form the electronically excited oxyluciferin anion that subsequently relaxes by emitting a photon of visible light. Although all bioluminescent beetle species use the same small molecule (D-luciferin) and the same reaction to produce light, the colour of the emission varies from green to red depending on the species, or in the case of click 


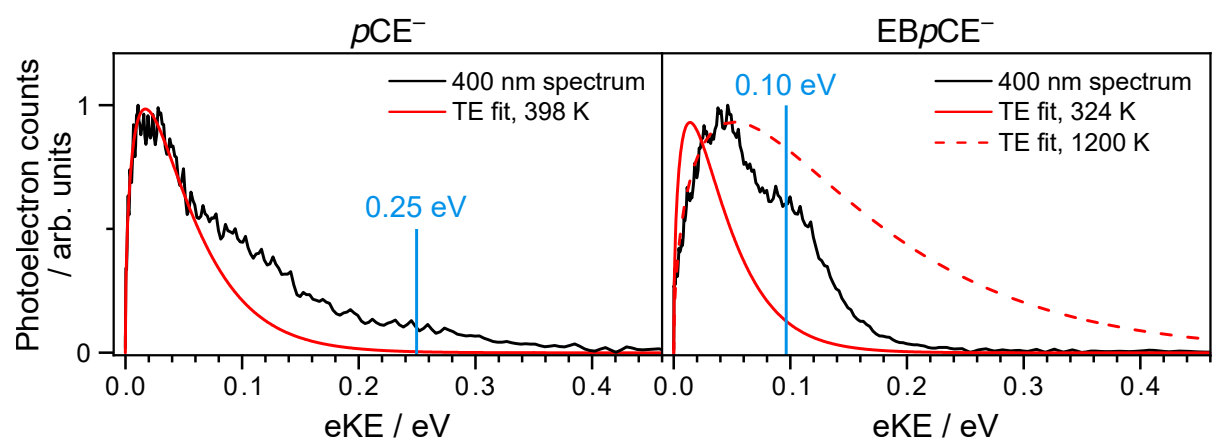

Figure 25. Photoelectron spectra of model chromophore $p \mathrm{CE}^{-}$(left) and isomerisation-locked chromophore $\mathrm{EB} p \mathrm{CE}^{-}$(right) recorded at $\sim 400 \mathrm{~nm}$ with intensities normalised to the maxima. Modelled eKE distributions using Eq. 3 at the temperatures specified; solid red lines employ $T_{\mathrm{M}} \cdot$ values estimated from quantum chemistry calculations and the dashed red line shows that an unphysical $T_{\mathrm{M}}$. of $>1000 \mathrm{~K}$ is required to fit the rising edge for $\mathrm{EB} p \mathrm{CE}^{-}$. Vertical blue lines mark the eKEs corresponding to the expected eKEs from direct photodetachment $(h \nu-V D E)$. Adapted with permission from [34]. Copyright 2018 American Chemical Society.

beetles, even between individuals. In order to understand how the environment of the luciferin tunes its electronic structure, there has been recent interest in understanding the intrinsic electronic structure and relaxation dynamics of the isolated luciferin and oxyluciferin molecules in the gas phase [30, 113, 114].

\subsection{Photoelectron spectroscopy of luciferin chromophores}

Using a combination of photoelectron spectroscopy measurements and EOM-IPCCSD/aug-cc-pVDZ and ADC(2)/aug-cc-pVDZ calculations of deprotonated luciferin anions, it was found that electrospray ionisation of luciferin in dry methanol solution generated, predominantly, anions in their phenolate forms (Fig. 28), which are the biologically relevant forms for gas-phase studies [30]. It was revealed that the minimum energy conformation of the isolated luciferin anion has the carboxylic acid group in the plane of the molecule, unlike the chromophore in the enzyme environment. In the gas phase, the planar conformation is stabilised by an intramolecular interaction between the acidic proton and the thiazole nitrogen. This is significant as it suggests that this interaction may not occur in the protein due to binding of the carboxylate oxygen to a nearby lysine residue and the distinct conformation of the carboxylic acid group in the enzyme could facilitate the attack of the phosphate group of ATP by a carboxylate oxygen atom in the adenylation step of the bioluminescence reaction.

Photoelectron spectra of luciferin were observed to broaden with increasing photon energy and it was found that this shoulder extended all the way to zero eKE, suggesting a transfer of energy from electronic into nuclear degrees of freedom (Fig. 28). ADC(2)/aug-cc-pVDZ calculations revealed that the closest bright electronically excited state, $\mathrm{S}_{3}$, had Feshbach resonance character with respect to the detachment continuum; thus it was concluded that the low eKE electrons were most likely attributed to internal conversion from the $\mathrm{S}_{3}$ state to the $\mathrm{S}_{1}$ state, which has shape 

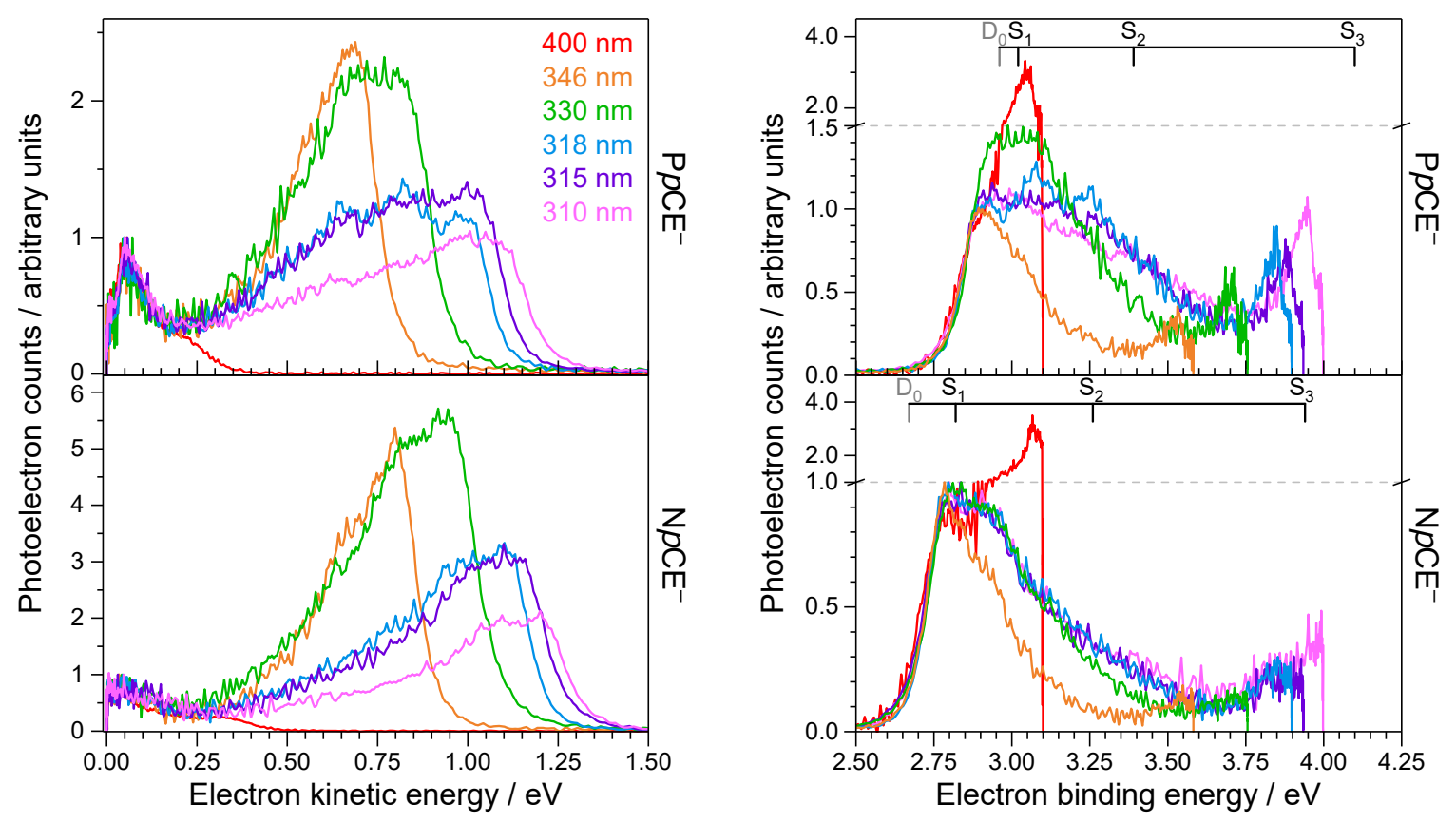

Figure 26. Photoelectron spectra of $\mathrm{P} p \mathrm{CE}^{-}$and $\mathrm{N} p \mathrm{CE}^{-}$recorded at wavelengths 310-400 $\mathrm{nm}$. Intensities of the spectra plotted as a function of eKE (left) have been normalised to the maximum of the low eKE feature. Intensities of the spectra plotted as a function of eBE (right) have been scaled to align the rising edges and are normalised to the maximum of the rising edges of the $346 \mathrm{~nm}$ spectra. Combs mark the EPT/6-311++G(3df,3pd) VDEs (grey) and the first three singlet excited states of the anion calculated using $\mathrm{ADC}(2) / 6-31+\mathrm{G}^{* *}$ (black). Adapted from Ref. [29] with permission from the PCCP Owner Societies.<smiles>COc1ccc2nc(C3=NC(C(=O)O)CS3)sc2c1</smiles>

Figure 27. Structure of the deprotonated luciferin anion.

resonance character with respect to the detachment continuum, followed by autodetachment. This hints at the possibility of new biological applications for this family of bioluminescent chromophores.

Interestingly, photoelectron spectra of deprotonated infraluciferin (Fig. 29), the furthest red-shifted analogue of luciferin, revealed a broadening on the low eBE side of the photoelectron spectra that was not observed in the photoelectron spectra of deprotonated luciferin (Fig. 30). The intensity of this feature was found to decrease with increasing photon energy, suggesting that it could arise from a fragmentation process following resonant electronic excitation of the chromophore anion. ADC(2)/aug-ccpVDZ and EOM-IP-CCSD/aug-cc-pVDZ calculations revealed that this feature was most likely attributed to resonant excitation of the $\mathrm{S}_{3}$ state of the anion and pho- 


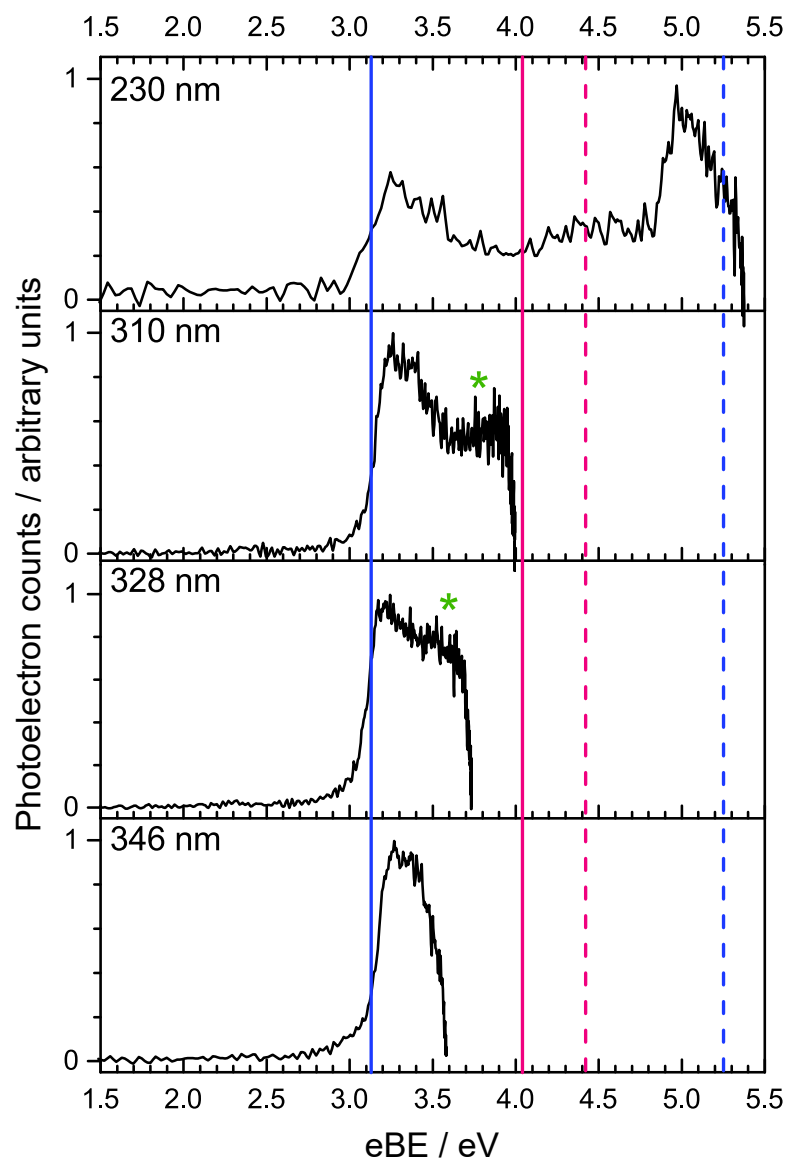

Figure 28. Photoelectron spectra of deprotonated luciferin anions. Vertical blue and pink lines mark the EOM-IP-CCSD calculated VDEs of the phenolate and carboxylate anions $\left(\mathrm{D}_{0}\right.$ solid, $\mathrm{D}_{1}$ dashed); these show that the anions are formed predominantly in their phenolate form in this experiment (electrospray ionisation from 1mM dry methanol solution). The asterisks mark the broadening on the high eBE edge of the peak that extends all the way to zero eKE. Reproduced from Ref. [30] with permission from the PCCP Owner Societies.

todissociation from this state, losing $\mathrm{CO}_{2}$.

\section{Summary and outlook}

There is increasing interest in employing anion photolectron spectroscopy to study deprotonated protein chromophores in the gas phase. Anion photoelectron spectroscopy provides a direct way of measuring electronic structure through the measurement of binding energies and, in the gas phase, provides direct access to the higher lying electronically excited states of protein chromophores that cannot be measured easily in their native environments because the UV absorption of the chromophore overlaps with the UV absorption of aromatic amino acid residues in the protein.

Recent photoelectron spectroscopy measurements have revealed that the first elec- 
<smiles>COc1ccc2nc(/C=C/C3=NC(C(=O)O)CS3)sc2c1</smiles>

Figure 29. Structure of the deprotonated infraluciferin anion.

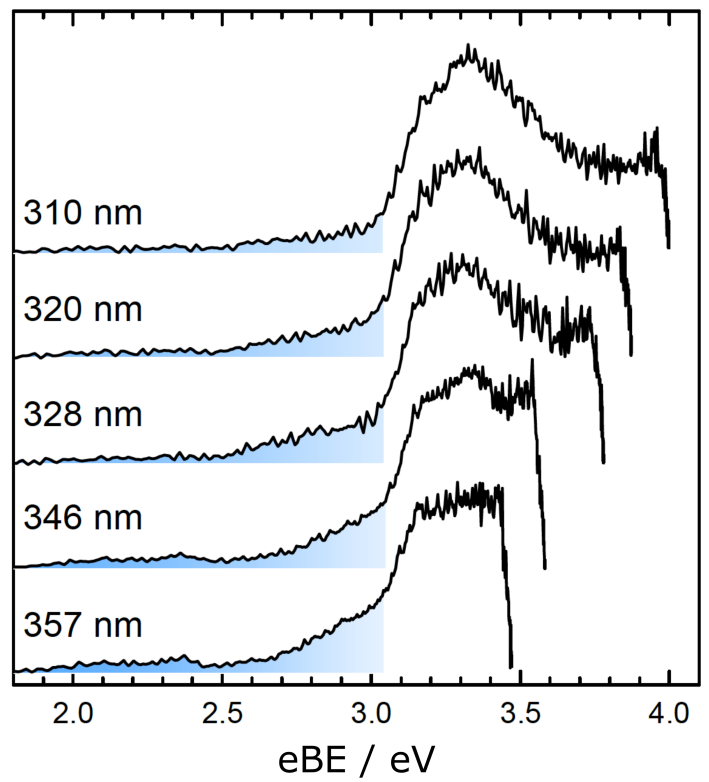

Figure 30. Photoelectron spectra of deprotonated infraluciferin anions. The blue shading highlights a broad feature on the low eBE edge of the direct detachment peak and additional features between $1.9 \mathrm{eV}$ and $2.4 \mathrm{eV}$ in the $357 \mathrm{~nm}$ and $346 \mathrm{~nm}$ spectra. The intensities of these spectra are normalised on the rising edges of the direct detachment peak, highlighting the wavelength dependence of the broadening. Reproduced from Ref. [30] with permission from the PCCP Owner Societies.

tronically excited states of the GFP and PYP protein chromophores in vacuo lie below the adiabatic detachment energies and have identified higher lying electronically excited states that are likely to be involved in decarboxylation reactions of GFP and the formation of solvated electrons in PYP. It has also been shown that it is possible to control the electronic structure and electronic relaxation dynamics by introducing chemical modifications such as electron-donating and electron-withdrawing substituents and structural 'locks' that control torsional motions. Time-resolved photoelectron spectroscopy measurements have revealed that the initial dynamics of the GFP and PYP protein chromophores in vacuo are similar to those in solution and that the influence of the protein manifests itself further away from the Franck-Condon region. Photoelectron spectroscopy studies of the luciferin chromophore revealed differences in the conformation in the gas phase and the enzyme that could be linked 
to the adenylation step of the bioluminescent reaction.

Promising future directions are likely to include high-resolution studies of cryogenically cooled chromophores and time-resolved studies of microsolvated chromophores to investigate the role of crystallographic water molecules or to mimic interactions with key residues in the protein cavity. These studies will not only provide new insights that may inform the rational design of new photoactive proteins with specific functions, but will serve as important benchmarks for the development of computational methods for treating resonances in the detachment continuum.

\section{Acknowledgements}

We thank the EPSRC for financial support and the Leverhulme Trust and the Royal Society for a Royal Society Leverhulme Trust Senior Research Fellowship (HHF). We thank Joanne Woodhouse for the $346 \mathrm{~nm}$ photoelectron spectrum of $p \mathrm{HBDI}^{-}$(Fig. 7 ) and many co-workers and collaborators for their contributions to work described in this review.

\section{References}

[1] B. Valeur and M. N. Berberan-Santos, Molecular Fluorescence Principles and Applications, Wiley-VCH, 2012.

[2] D. Hu, L. Yao, B. Yang and Y. Ma, Phil. Trans. R. Soc. A., 2015, 373, 20140318.

[3] R. Y. Tsien, Annu. Rev. Biochem., 1998, 67, 509-544.

[4] M. Zimmer, Chem. Rev., 2002, 102, 759-782.

[5] K. J. Hellingwerf, J. Hendriks and T. Gensch, J. Phys. Chem. A, 2003, 107, 1082 1094.

[6] K. Niwa, Y. Ichino and Y. Ohmiya, Chem. Lett., 2010, 39, 291-293.

[7] W. Lee, G. Kodali, R. J. Stanley and S. Matsika, Chem. Eur. J., 2016, 22, 1137111381.

[8] Images of 2WUR: A. Shinobu, G. J. Palm, A. J. Schierbeek and N. Agmon, J. Am. Chem. Soc., 2010, 132, 11093-11102; 1NWZ: E. D. Getzoff, K. N. Gutwin and U. K. Genick, Nat. Struct. Biol., 2003, 10, 663-668; and 2D1R: T. Nakatsu, S. Ichiyama, J. Hiratake, A. Saldanha, N. Kobashi, K. Sakata and H. Kato, Nature, 2006, 440, 372-376. Created with VMD - Visual Molecular Dynamics (W. Humphrey, A. Dalke and K. Schulten, J. Molec. Graphics, 1996, 14, 33-38).

[9] T. Koopmans, Physica, 1934, 1, 104-113.

[10] C. L. Adams, H. Schneider and J. M. Weber, J. Phys. Chem. A, 2010, 114, 4017-4030.

[11] C. E. Klots, J. Chem. Phys., 1993, 98, 1110-1115.

[12] C. E. Klots, J. Chem. Phys., 1994, 100, 1035-1039.

[13] R. Mabbs, E. Surber and A. Sanov, Analyst, 2003, 128, 765-772.

[14] J. B. Wills, F. Pagliarulo, B. Baguenard, F. Lépine and C. Bordas, Chem. Phys. Lett., 2004, 390, 145-150.

[15] B. Climen, F. Pagliarulo, A. Ollagnier, B. Baguenard, B. Concina, M. A. Lebeault, F. Lépine and C. Bordas, Eur. Phys. J. D, 2007, 43, 85-89.

[16] E. P. Wigner, Phys. Rev., 1948, 73, 1002-1009.

[17] U. Fano and D. Dill, Phys. Rev. A, 1973, 6, 185-192.

[18] A. R. McKay, M. E. Sanz, C. R. S. Mooney, R. S. Minns, E. M. Gill and H. H. Fielding, Rev. Sci. Instrum., 2010, 81, 123101.

[19] C. R. S. Mooney, M. E. Sanz, A. R. McKay, R. J. Fitzmaurice, A. E. Aliev, S. Caddick and H. H. Fielding, J. Phys. Chem. A, 2012, 116, 7943-7949.

[20] D. A. Horke and J. R. R. Verlet, Phys. Chem. Chem. Phys., 2012, 14, 8511-8515. 
[21] Y. Toker, D. B. Rahbek, B. Klærke, A. V. Bochenkova and L. H. Andersen, Phys. Rev. Lett., 2012, 109, 128101.

[22] C. R. S. Mooney, D. A. Horke, A. S. Chatterley, A. Simperler, H. H. Fielding and J. R. R. Verlet, Chem. Sci., 2013, 4, 921-927.

[23] C. R. S. Mooney, M. A. Parkes, L. Zhang, H. C. Hailes, A. Simperler, M. J. Bearpark and H. H. Fielding, J. Chem. Phys., 2014, 140, 205103.

[24] S. H. M. Deng, X.-Y. Kong, G. Zhang, Y. Yang, W.-J. Zheng, Z.-R. Sun, D.-Q. Zhang and X.-B. Wang, J. Phys. Chem. Lett., 2014, 5, 2155-2159.

[25] C. R. S. Mooney, M. A. Parkes, A. Iskra and H. H. Fielding, Angew. Chem. Int. Ed., 2015, 54, 5646-5649.

[26] C. W. West, J. N. Bull, A. S. Hudson, S. L. Cobb and J. R. R. Verlet, J. Phys. Chem. $B, 2015, \mathbf{1 1 9}, 3982-3987$.

[27] K. Bhaskaran-Nair, M. Valiev, S. H. M. Deng, W. A. Shelton, K. Kowalski and X.-B. Wang, J. Chem. Phys., 2015, 143, 224301.

[28] M. A. Parkes, C. Phillips, M. J. Porter and H. H. Fielding, Phys. Chem. Chem. Phys., 2016, 18, 10329-10336.

[29] A. Henley, M. E. Diveky, A. M. Patel, M. A. Parkes, J. C. Anderson and H. H. Fielding, Phys. Chem. Chem. Phys., 2017, 19, 31572-31580.

[30] J. L. Woodhouse, M. Assmann, M. A. Parkes, H. Grounds, S. J. Pacman, J. C. Anderson, G. A. Worth and H. H. Fielding, Phys. Chem. Chem. Phys., 2017, 19, 22711-22720.

[31] A. V. Bochenkova, C. R. S. Mooney, M. A. Parkes, J. L. Woodhouse, L. Zhang, R. Lewin, J. M. Ward, H. C. Hailes, L. H. Andersen and H. H. Fielding, Chem. Sci., 2017, 8, 3154-3163.

[32] J. Tay, M. A. Parkes, K. Addison, Y. Chan, L. Zhang, H. C. Hailes, P. C. Bulman Page, S. R. Meech, L. Blancafort and H. H. Fielding, J. Phys. Chem. Lett., 2017, 8, $765-771$.

[33] C. McLaughlin, M. Assmann, M. A. Parkes, J. L. Woodhouse, R. Lewin, H. C. Hailes, G. A. Worth and H. H. Fielding, Chem. Sci., 2017, 8, 1621-1630.

[34] A. Henley, A. M. Patel, M. A. Parkes, J. C. Anderson and H. H. Fielding, J. Phys. Chem. A, 2018, 122, 8222-8228.

[35] M. A. Parkes, J. Crellin, A. Henley and H. H. Fielding, Phys. Chem. Chem. Phys., 2018, 20, 15543-15549.

[36] P. Kruit and F. H. Read, J. Phys. E: Sci. Instrum., 1983, 16, 313-324.

[37] A. T. J. B. Eppink and D. H. Parker, Rev. Sci. Instrum., 1997, 68, 3477-3484.

[38] A. Sanov, Annu. Rev. Phys. Chem., 2014, 65, 341-363.

[39] C. S. Anstöter, J. N. Bull and J. R. Verlet, Int. Rev. Phys. Chem., 2016, 35, 509-538.

[40] C. S. Anstöter, C. R. Dean and J. R. Verlet, J. Phys. Chem. Lett., 2017, 8, 2268-2273.

[41] A. D. Becke, J. Chem. Phys., 1993, 98, 5648-5652.

[42] C. Lee, W. Yang and R. G. Parr, Phys. Rev. B, 1988, 37, 785-789.

[43] S. H. Vosko, L. Wilk and M. Nusair, Can. J. Phys., 1980, 58, 1200-1211.

[44] P. J. Stephens, F. J. Devlin, C. F. Chabalowski and M. J. Frisch, J. Phys. Chem., 1994, 98, 11623-11627.

[45] T. Yanai, D. P. Tew and N. C. Handy, Chem. Phys. Lett., 2004, 393, 51-57.

[46] R. Krishnan, J. S. Binkley, R. Seeger and J. A. Pople, J. Chem. Phys., 1980, 72, 650-654.

[47] T. Clark, J. Chandrasekhar, G. W. Spitznagel and P. V. R. Schleyer, J. Comput. Chem., 1983, 4, 294-301.

[48] M. J. Frisch, J. A. Pople and J. S. Binkley, J. Chem. Phys., 1984, 80, 3265-3269.

[49] R. A. Kendall, T. H. Dunning Jr and R. J. Harrison, J. Chem. Phys., 1992, 96, 6796-6806.

[50] J. Linderberg and Y. Öhrn, Propagators in Quantum Chemistry, John Wiley \& Sons, Inc., Hoboken, NJ, USA, 2004, p. 79.

[51] V. G. Zakrzewski, O. Dolgounitcheva, A. V. Zakjevskii and J. V. Ortiz, Annu. Rep. Comput. Chem., 2010, 6, 79-94.

[52] A. I. Krylov, Annu. Rev. Phys. Chem., 2008, 59, 433-462. 
[53] R. Beekmeyer, M. A. Parkes, L. Ridgewell, J. W. Riley, B. L. Feringa, A. Kerridge and H. H. Fielding, Chem. Sci., 2017, 8, 6141-6148.

[54] E. Runge and E. K. Gross, Phys. Rev. Lett., 1984, 52, 997-1000.

[55] J. Schirmer, Phys. Rev. A, 1982, 26, 2395-2416.

[56] A. B. Trofimov and J. Schirmer, J. Phys. B At. Mol. Opt. Phys., 1995, 28, 2299-2324.

[57] F. F. García-Prieto, M. A. Aguilar, I. F. Galván, A. Muñoz-Losa, F. J. Olivares del Valle, M. L. Sánchez and M. E. Martín, J. Phys. Chem. A, 2015, 119, 5504-5514.

[58] T.-C. Jagau, K. B. Bravaya and A. I. Krylov, Annu. Rev. Phys. Chem., 2017, 68, $525-553$.

[59] T. Rocha-Rinza, O. Christiansen, J. Rajput, A. Gopalan, D. B. Rahbek, L. H. Andersen, A. V. Bochenkova, A. A. Granovsky, K. B. Bravaya, A. V. Nemukhin, K. L. Christiansen and M. B. Nielsen, J. Phys. Chem. A, 2009, 113, 9442-9449.

[60] V.A. Mozhayskiy and A.I. Krylov, ezSpectrum, http://iopenshell.usc.edu/downloads.

[61] S. Gozem and A.I. Krylov, ezDyson, http://iopenshell.usc.edu/downloads/ezdyson.

[62] C. M. Oana and A. I. Krylov, J. Chem. Phys., 2007, 127, 234106.

[63] J. V. Ortiz, WIREs Comput. Mol. Sci., 2013, 3, 123-142.

[64] S. R. Meech, Chem. Soc. Rev., 2009, 38, 2922-2934.

[65] A. S. Mishin, V. V. Belousov, K. M. Solntsev and K. A. Lukyanov, Curr. Opin. Chem. Biol., 2015, 27, 1-9.

[66] A. Acharya, A. M. Bogdanov, B. L. Grigorenko, K. B. Bravaya, A. V. Nemukhin, K. A. Lukyanov and A. I. Krylov, Chem. Rev., 2017, 117, 758-795.

[67] M. Chattoraj, B. A. King, G. U. Bublitz and S. G. Boxer, Proc. Natl. Acad. Sci. U. S. A., 1996, 93, 8362-8367.

[68] H. Niwa, S. Inouye, T. Hirano, T. Matsuno, S. Kojima, M. Kubota, M. Ohashi and F. Tsuji, Proc. Natl. Acad. Sci. U. S. A., 1996, 93, 13617-13622.

[69] N. M. Webber, K. L. Litvinenko and S. R. Meech, J. Phys. Chem. B, 2001, 105, 8036-8039.

[70] M. W. Forbes and R. A. Jockusch, J. Am. Chem. Soc., 2009, 131, 17038-17039.

[71] A. Svendsen, H. V. Kiefer, H. B. Pedersen, A. V. Bochenkova and L. H. Andersen, J. Am. Chem. Soc., 2017, 139, 8766-8771.

[72] A. Baldridge, S. R. Samanta, N. Jayaraj, V. Ramamurthy and L. M. Tolbert, J. Am. Chem. Soc, 2011, 133, 712-715.

[73] A. Baldridge, S. Feng, Y. T. Chang and L. M. Tolbert, ACS Comb. Sci., 2011, 13, 214-217.

[74] K. D. Warner, M. C. Chen, W. Song, R. L. Strack, A. Thorn, S. R. Jaffrey and A. R. Ferré-D'Amaré, Nat. Struct. Mol. Biol., 2014, 21, 658-663.

[75] E. A. Dolgopolova, A. M. Rice, M. D. Smith and N. B. Shustova, Inorg. Chem., 2016, 55, 7257-7264.

[76] D. Mandal, T. Tahara and S. R. Meech, J. Phys. Chem. B, 2004, 108, 1102-1108.

[77] M. E. Martin, F. Negri and M. Olivucci, J. Am. Chem. Soc., 2004, 126, 5452-5464.

[78] A. Acharya, A. M. Bogdanov, B. L. Grigorenko, K. B. Bravaya, A. V. Nemukhin, K. A. Lukyanov and A. I. Krylov, Chem. Rev., 2017, 117, 758-795.

[79] S. B. Nielsen, A. Lapierre, J. U. Andersen, U. V. Pedersen, S. Tomita and L. H. Andersen, Phys. Rev. Lett., 2001, 87, 228102.

[80] E. Epifanovsky, I. Polyakov, B. Grigorenko, A. Nemukhin and A. I. Krylov, J. Chem. Theory Comput., 2009, 5, 1895-1906.

[81] K. B. Bravaya and A. I. Krylov, J. Phys. Chem. A, 2013, 117, 11815-22.

[82] H. H. Fielding and G. A. Worth, Chem. Soc. Rev., 2018, 47, 309-321.

[83] J. J. van Thor, Chem. Soc. Rev., 2009, 38, 2935-2950.

[84] J. S. Paige, K. Y. Wu and S. R. Jaffrey, Science, 2011, 333, 642-6.

[85] R. Ando, H. Hama, M. Yamamoto-Hino, H. Mizuno and A. Miyawaki, Proc. Natl. Acad. Sci. U. S. A., 2002, 99, 12651-12656.

[86] W. D. Hoff, P. Düx, K. Hård, B. Devreese, I. M. Nugteren-Roodzant, W. Crielaard, R. Boelens, R. Kaptein, J. Van Beeumen and K. J. Hellingwerf, Biochemistry, 1994, 33, 13959-13962.

[87] R. Brudler, R. Rammelsberg, T. T. Woo, E. D. Getzoff and K. Gerwert, Nat. Struct. 
Biol., 2001, 8, 265-270.

[88] M. Baca, G. E. Borgstahl, M. Boissinot, P. M. Burke, D. W. R. Williams, K. A. Slater and E. D. Getzoff, Biochemistry, 1994, 33, 14369-14377.

[89] T. E. Meyer, E. Yakali, M. A. Cusanovich and G. Tollin, Biochemistry, 1987, 26, 418-423.

[90] T. E. Meyer, G. Tollin, J. H. Hazzard and M. A. Cusanovich, Biophys. J., 1989, 56, $559-564$.

[91] I. B. Nielsen, S. Boyé-Péronne, M. O. A. El Ghazaly, M. B. Kristensen, S. Brøndsted Nielsen and L. H. Andersen, Biophys. J., 2005, 89, 2597-2604.

[92] I.-R. Lee, W. Lee and A. H. Zewail, Proc. Natl. Acad. Sci. U. S. A., 2006, 103, 258-262.

[93] T. Rocha-Rinza, O. Christiansen, D. B. Rahbek, B. Klærke, L. H. Andersen, K. Lincke and M. Brøndsted Nielsen, Chem. Eur. J., 2010, 16, 11977-11984.

[94] S. Smolarek, A. Vdovin, D. L. Perrier, J. P. Smit, M. Drabbels and W. J. Buma, J. Am. Chem. Soc., 2010, 132, 6315-6317.

[95] M. Almasian, J. Grzetic, J. Van Maurik, J. D. Steill, G. Berden, S. Ingemann, W. J. Buma and J. Oomens, J. Phys. Chem. Lett., 2012, 3, 2259-2263.

[96] F. F. García-Prieto, I. F. Galván, A. Muñoz-Losa, M. A. Aguilar and M. E. Martín, J. Chem. Theory Comput., 2013, 9, 4481-4494.

[97] M. E. van Brederode, T. Gensch, W. D. Hoff, K. J. Hellingwerf and S. E. Braslavsky, Biophys. J., 1995, 68, 1101-1109.

[98] U. K. Genick, S. M. Soltis, P. Kuhn, I. L. Canestrelli and E. D. Getzoff, Nature, 1998, 392, 206-209.

[99] L. J. G. W. van Wilderen, M. A. van der Horst, I. H. M. van Stokkum, K. J. Hellingwerf, R. van Grondelle and M. L. Groot, Proc. Natl. Acad. Sci. U. S. A., 2006, 103, 15050-15055.

[100] P. D. Coureux, Z. P. Fan, V. Stojanoff and U. K. Genick, Structure, 2008, 16, 863-872.

[101] G. Groenhof, L. V. Schäfer, M. Boggio-Pasqua, H. Grubmüller and M. A. Robb, J. Am. Chem. Soc., 2008, 130, 3250-3251.

[102] G. Groenhof, M. Bouxin-Cademartory, B. Hess, S. P. de Visser, H. J. C. Berendsen, M. Olivucci, A. E. Mark and M. A. Robb, J. Am. Chem. Soc., 2004, 126, 4228-4233.

[103] D. S. Larsen, M. Vengris, I. H. van Stokkum, M. A. van der Horst, F. L. de Weerd, K. J. Hellingwerf and R. van Grondelle, Biophys. J., 2004, 86, 2538-2550.

[104] P. Changenet-Barret, P. Plaza and M. M. Martin, Chem. Phys. Lett., 2001, 336, 439-444.

[105] J. Zhu, L. Paparelli, M. Hospes, J. Arents, J. T. M. Kennis, I. H. M. van Stokkum, K. J. Hellingwerf and M. L. Groot, J. Phys. Chem. B, 2013, 117, 11042-11048.

[106] D. S. Larsen, I. H. M. van Stokkum, M. Vengris, M. A. van der Horst, F. L. de Weerd, K. J. Hellingwerf and R. van Grondelle, Biophys. J., 2004, 87, 1858-1872.

[107] C. N. Lincoln, A. E. Fitzpatrick and J. J. Thor, Phys. Chem. Chem. Phys., 2012, 14, 15752-15764.

[108] E. V. Gromov, I. Burghardt, J. T. Hynes, H. Köppel and L. S. Cederbaum, J. Photochem. Photobiol. A Chem., 2007, 190, 241-257.

[109] E. V. Gromov, J. Chem. Phys., 2014, 141, 224308-224308.

[110] F. F. García-Prieto, A. Muñoz-Losa, I. Fdez. Galván, M. L. Sánchez, M. A. Aguilar and M. E. Martín, J. Chem. Theory Comput., 2017, 13, 737-748.

[111] M. Boggio-Pasqua and G. Groenhof, J. Phys. Chem. B, 2011, 115, 7021-7028.

[112] C. Ko, A. M. Virshup and T. J. Martínez, Chem. Phys. Lett., 2008, 460, 272-277.

[113] K. Støchkel, B. F. Milne and S. Brøndsted Nielsen, J. Phys. Chem. A, 2011, 115, $2155-9$.

[114] K. Støchkel, C. Nygaard Hansen, J. Houmøller, L. Munksgaard Nielsen, K. Anggara, M. Linares, P. Norman, F. Nogueira, O. V. Maltsev, L. Hintermann, S. Brøndsted Nielsen, P. Naumov and B. F. Milne, J. Am. Chem. Soc., 2013, 135, 6485-6493. 